\title{
Myo-inositol oxygenase expression profile modulates pathogenic ferroptosis in the renal proximal tubule
}

\author{
Fei Deng, ${ }^{1,2}$ Isha Sharma, ${ }^{2}$ Yingbo Dai, ${ }^{3}$ Ming Yang, ${ }^{4}$ and Yashpal S. Kanwar ${ }^{2}$ \\ 'Department of Urology, The Third Xiangya Hospital, Central South University, Changsha, Hunan, China. ${ }^{2}$ Department of Pathology \& Medicine, Northwestern University, Chicago, Illinois, USA. ${ }^{3}$ Department \\ of Urology, The Fifth Affiliated Hospital of Sun Yet-Sen University, Zhuhai, Guangdong, China. ${ }^{4}$ Department of Nephrology, The Second Xiangya Hospital, Central South University, Changsha, Hunan, China.
}

\begin{abstract}
Overexpression of myo-inositol oxygenase (MIOX), a proximal tubular enzyme, exacerbates cellular redox injury in acute kidney injury (AKI). Ferroptosis, a newly coined term associated with lipid hydroperoxidation, plays a critical role in the pathogenesis of AKI. Whether or not MIOX exacerbates tubular damage by accelerating ferroptosis in cisplatin-induced AKI remains elusive. Cisplatin-treated HK-2 cells exhibited notable cell death, which was reduced by ferroptosis inhibitors. Also, alterations in various ferroptosis metabolic sensors, including lipid hydroperoxidation, glutathione peroxidase 4 (GPX4) activity, NADPH and reduced glutathione (CSH) levels, and ferritinophagy, were observed. These perturbations were accentuated by MIOX overexpression, while ameliorated by MIOX knockdown. Likewise, cisplatin-treated CD1 mice exhibited tubular damage and derangement of renal physiological parameters, which were alleviated by ferrostatin-1, a ferroptosis inhibitor. To investigate the relevance of MIOX to ferroptosis, WT mice, MIOX-overexpressing transgenic (MIOX-Tg) mice, and MIOX-KO mice were subjected to cisplatin treatment. In comparison with cisplatin-treated WT mice, cisplatin-treated MIOX-Tg mice had more severe renal pathological changes and perturbations in ferroptosis metabolic sensors, which were minimal in cisplatin-treated MIOX-KO mice. In conclusion, these findings indicate that ferroptosis, an integral process in the pathogenesis of cisplatin-induced AKI, is modulated by the expression profile of MIOX.
\end{abstract}

\section{Introduction}

Acute kidney injury (AKI) is characterized by a rapid deterioration of renal functions, and it presents as one of the major causes of morbidity in hospitalized patients $(1,2)$. It is estimated that more than 2 million deaths a year are related to AKI around the world. Also, in the United States, AKI is a great burden to the health care system in terms of management and dollar cost $(3,4)$. Those who survive AKI have a high risk of progressing to chronic kidney disease or even end-stage renal disease $(5,6)$. Clinically, AKI can occur in a wide variety of clinicopathological states, including ischemia/reperfusion injury (IRI), sepsis, and the administration of various nephrotoxic agents (7). The pathophysiology of AKI is complex, involving various cell types and multiple factors and signaling events related to regulated cell death (7). Morphologically, AKI is characterized by acute tubular necrosis (ATN), renal inflammation, glomerular injury, and vascular dysfunction (7). One of the agents that commonly induce AKI is cisplatin, a nephrotoxic agent widely used in the treatment of patients with various forms of neoplasms. Cisplatin-induced nephropathy has been extensively investigated in various animal models to explore the pathogenesis of AKI. Previous studies have shown that cisplatin is reabsorbed from the glomerular ultrafiltrate in proximal tubules, and it leads to severe ATN due to the DNA damage, cellular redox

Conflict of interest: The authors have declared that no conflict of interest exists. Copyright: @ 2019, American Society for Clinical Investigation.

Submitted: April 29, 2019; Accepted: August 16, 2019; Published: October 14, 2019

Reference information: J Clin Invest. 2019;129(11):5033-5049.

https://doi.org/10.1172/JCl129903. imbalance, and mitochondrial dysfunctions (7, 8). Recently, cisplatin was noted to induce death of cancer cells via different processes, including ferroptosis (9).

In 2012, a new term for regulated cell death was coined: ferroptosis, characterized by intracellular accumulation of lipid hydroperoxides and overload of free iron (9). The process of ferroptosis is independent of molecular pathways that involve caspase and necrosomal complex, while it can be terminated by glutathione peroxidase 4 (GPX4) (9). It has been reported that ferroptosis is incriminated in the pathogenesis of many diseases, such as Huntington's disease, Parkinson's disease, hemochromatosis, urinary tract infections, and death of neoplastic cells following chemotherapy (9-11). Owing to the abundance of polyunsaturated fatty acids in renal tubular cells (8), it is conceivable that the kidney is readily susceptible to lipid hydroperoxide-induced ferroptosis. In fact, it has been shown that the process of ferroptosis is more relevant than necroptosis and apoptosis in IRI-induced ATN, and it is also the primary cause of folic acid-induced AKI $(9,12)$, but its role in cisplatin-induced AKI remains to be explored.

Myo-inositol oxygenase (MIOX), a renal proximal tubularspecific enzyme, was identified several years ago (13). It catabolizes myo-inositol to D-glucuronate, and plays an important role in renal tubular injury (14). The MIOX promoter includes osmotic response elements, carbohydrate response elements, oxidant response elements, and sterol response elements, and thus MIOX transcription is modulated by oxidant stress, high glucose ambience, and the administration of free fatty acids $(8,14,15)$. Besides, epigenetic studies have revealed that MIOX expression is depen- 

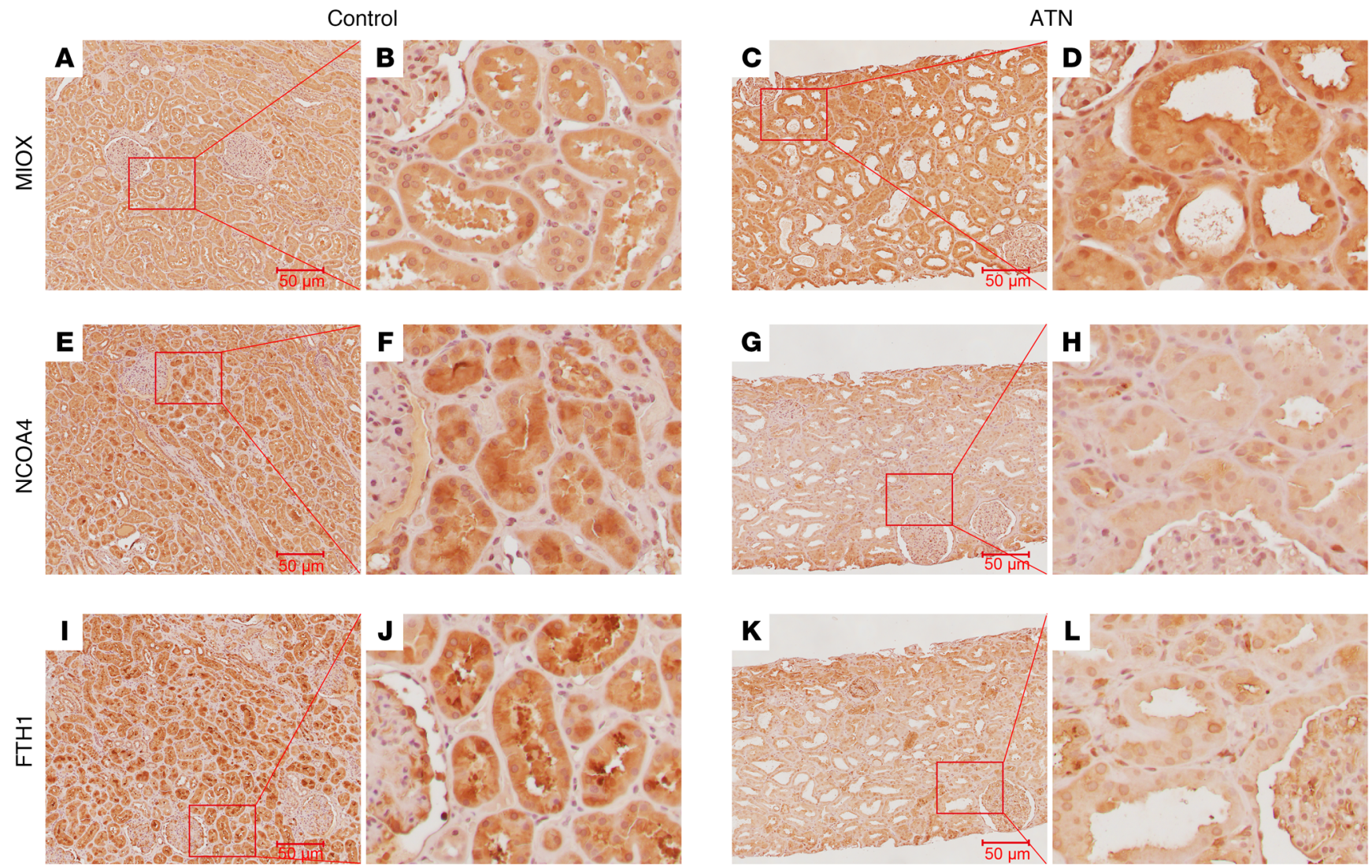

Figure 1. Expression of MIOX and ferritinophagy markers in kidneys of patients with ATN, as assessed by immunohistochemistry. Scanning-magnification photomicrographs revealed that MIOX was expressed in the proximal tubular cells, and its expression was increased notably in patients with ATN, as readily seen in high-magnification photomicrographs ( $\mathbf{C}$ and $\mathbf{D}$ vs. A and B). No MIOX expression was seen in the glomerulus. The ferritinophagy biomarkers NCOA4 (cargo receptor of ferritin) and FTH1 (heavy chain of ferritin) were seen localized in the renal tubular epithelia, and their expression was notably decreased in patients with ATN ( $\mathbf{G}$ and $\mathbf{H}$ vs. $\mathbf{E}$ and $\mathbf{F}$, and $\mathbf{K}$ and $\mathbf{L}$ vs. I and $\mathbf{~ )}$ ). Like MIOX, no significant expression of ferritinophagy markers was noted in the glomerular compartment. Scale bars: $50 \mu \mathrm{m}$. Panels $\mathrm{G}$ and $\mathrm{K}$ are from consecutive sections from the same patient sample but stained with different antibodies.

dent on demethylation of MIOX promoter in a diabetic state or cisplatin-induced AKI $(8,14)$. Upregulated MIOX expression also promotes the generation of reactive oxygen species (ROS), and thus it conceivably exacerbates renal tubular injury in a variety of pathological states. However, its relevance to the process of ferroptosis remains to be investigated. Our exploratory studies on kidney biopsy tissues of patients with ATN revealed an increased expression of MIOX in the proximal tubular epithelium (Figure $1, \mathrm{C}$ and D vs. A and B). Concomitantly, there was a decreased expression of ferritinophagy biomarkers, i.e., nuclear receptor coactivator 4 (NCOA4) and heavy chain of ferritin (FTH1), in the renal tubular epithelia of these patients (Figure 1, G and $\mathrm{H}$ vs. E and $\mathrm{F}$, and $\mathrm{K}$ and $\mathrm{L}$ vs. I and J). Because of these changes in the temporal expression of MIOX and ferritinophagy markers in the renal tubular compartment, we investigated the pathobiology of MIOX and ferroptosis in cisplatin-induced AKI.

\section{Results}

The observations described below suggest that one important mechanism leading to AKI is mediated by a recently described pathobiological process, known as ferroptosis, in addition to apoptosis and necroptosis. In this process, a multitude of signaling molecules and factors may be involved that ultimately lead to cell death, of which ferroptosis seems to be an integral part as described below in the cisplatin-induced AKI model.

Ferroptosis is an essential part of cisplatin-induced HK-2 cell death. Normally, the HK-2 cells yielded a flat epithelial morphology in cultures maintained for 48 hours, as indicated by phase-contrast microscopy and direct visualization of $\mathrm{H} \& \mathrm{E}$ staining (Figure 2, A and D, red arrow and arrowhead). Cisplatin treatment led to contraction or shrinkage of HK-2 cells (Figure 2, B and E, yellow arrows and arrowheads), while cotreatment with cisplatin and ferrostatin-1 (Fer-1; an inhibitor of ferroptosis) partially reversed the morphological changes (Figure 2, C and F, blue arrow and arrowhead). TUNEL staining revealed notable DNA damage in cisplatin-treated HK-2 cells compared with the control cells (Figure 2, H vs. G). The cotreatment with Fer-1 remarkably reduced the degree of cell death (Figure 2I). Cell viability, as judged by MTT assay, was substantially decreased with cisplatin treatment, which could be rescued by concomitant treatment with Fer-1 (Figure 2J). Interestingly, treatment with deferoxamine (an iron chelator) and Z-VAD(OMe)-FMK (an apoptosis inhibitor) also led to increased survival of the cells (Figure 2, J and K). However, treatment with necrostatin-1 (Nec-1; a necroptosis inhibitor) did not restore the viability of cells treated with cisplatin (Fig- 

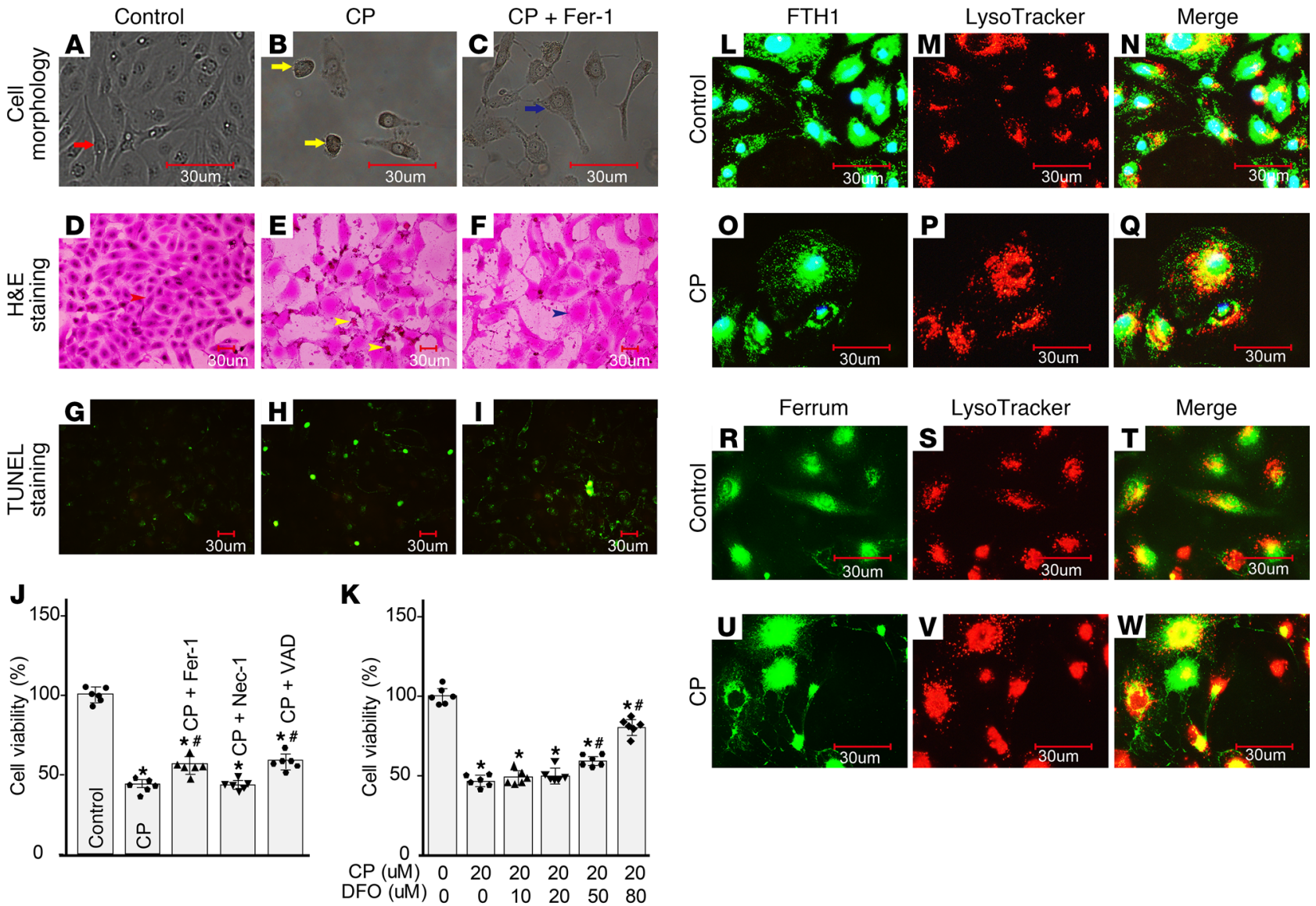

Figure 2. Ferroptosis is an essential part of cisplatin-induced HK-2 cell death. Normally, the HK-2 cells exhibited a flat epithelial morphology, demonstrated by phase-contrast microscopy and direct visualization of H\&E staining ( $\mathbf{A}$ and $\mathbf{D}$, red arrow and arrowhead). Cisplatin (CP) treatment caused shrinkage of HK-2 cells (B and E, yellow arrows and arrowheads), while cotreatment of cisplatin and Fer-1 led to a reversion of their morphology (C and $\mathbf{F}$, blue arrow and arrowhead). TUNEL staining revealed notable DNA damage in cisplatin-treated HK-2 cells, and cotreatment with Fer-1 reduced the cell death (G-I). MTT assay revealed reduced cell viability following cisplatin exposure, which was rescued by Fer- 1 treatment $(J)\left(n=6\right.$; ${ }^{*} P<0.05$ compared with the control group, ${ }^{\#} P<0.05$ compared with the CP group, 1-way ANOVA with Dunn's multiple comparisons). Deferoxamine (DFO) and Z-VAD(OMe)-FMK (VAD) treatment also led to an increased survival of cells, while Nec-1 was ineffective (J and $\mathbf{K})\left(n=6\right.$; ${ }^{*} P<0.05$ compared with the control group, ${ }^{\#} P<0.05$ compared with the CP group, 1-way ANOVA with Dunn's multiple comparisons). To investigate the intracellular dynamics of iron in HK-2 cells following cisplatin treatment, distribution of ferrum and heavy chain of ferritin (FTH1) was assessed. FTH1 was found distributed diffusely in the cytoplasm (green) and minimally in the Iysosomes (red) (L-N). Interestingly, FTH1 was seen heavily colocalized in the lysosomes following cisplatin treatment (0-Q). The ferrum was also found to be marginally codistributed within the lysosomes, and the codistribution markedly increased following cisplatin treatment (R-W). Scale bars: $30 \mu \mathrm{m}$.

ure $2 \mathrm{~J}$ ), suggesting that among these 3 processes, ferroptosis and apoptosis, but not necroptosis, are important modulators in cisplatin-induced HK-2 cell death. To emphasize the role of ferroptosis, we proceeded to investigate the intracellular dynamics of iron, specifically the ferrum- and iron-binding protein heavy chain of ferritin (FTH1). Normally, FTH1 (FITC-conjugated antibody) was found diffusely distributed in the cytoplasm and minimally localized in the lysosomes (LysoTracker Red), as evidenced by fluorescent microscopy (Figure 2, L-N), while FTH1 was seen abundantly colocalized in the lysosomes following cisplatin treatment (Figure 2, O-Q), which could be readily appreciated in the merged photographs (Figure 2, Q vs. $\mathrm{N})$. Along similar lines, ferrum was found to be mildly to moderately codistributed with lysosomes; however, their codistribution markedly increased following cisplatin treatment (Figure 2, $\mathrm{R}-\mathrm{W})$. Overall, these initial in vitro studies suggested that the pathobiology of ferrum and lysosomes seems to be intertwined during the process of ferroptosis induced by cisplatin, and this led us to investigate its biology in in vivo model systems.

Ferroptosis inhibition attenuates cisplatin-induced AKI. To elucidate the role of ferroptosis in cisplatin-induced AKI, Fer-1 was administered i.p. to CD1 mice about 45 minutes before cisplatin treatment. The renal morphological changes in cisplatin-treated mice included degeneration of tubular epithelia with loss of brush borders and dilatation (Figure 3, B vs. A). Many of the tubules included casts in the tubular lumina. These tubular changes were considerably attenuated by the administration of Fer-1 (Figure 3, $\mathrm{C}$ vs. B). No discernible changes were observed in the glomerular compartment. Periodic acid-Schiff (PAS) staining also revealed tubular epithelial disruption with sloughing off of the epithelia and shedding of PAS-positive material in the tubular lumina (Figure 3, E vs. D). Interestingly, these changes were largely reversed in mice that received prior treatment with Fer-1 (Figure 3, F vs. E). Urinary albumin excretion, assessed by SDS-PAGE, was increased 

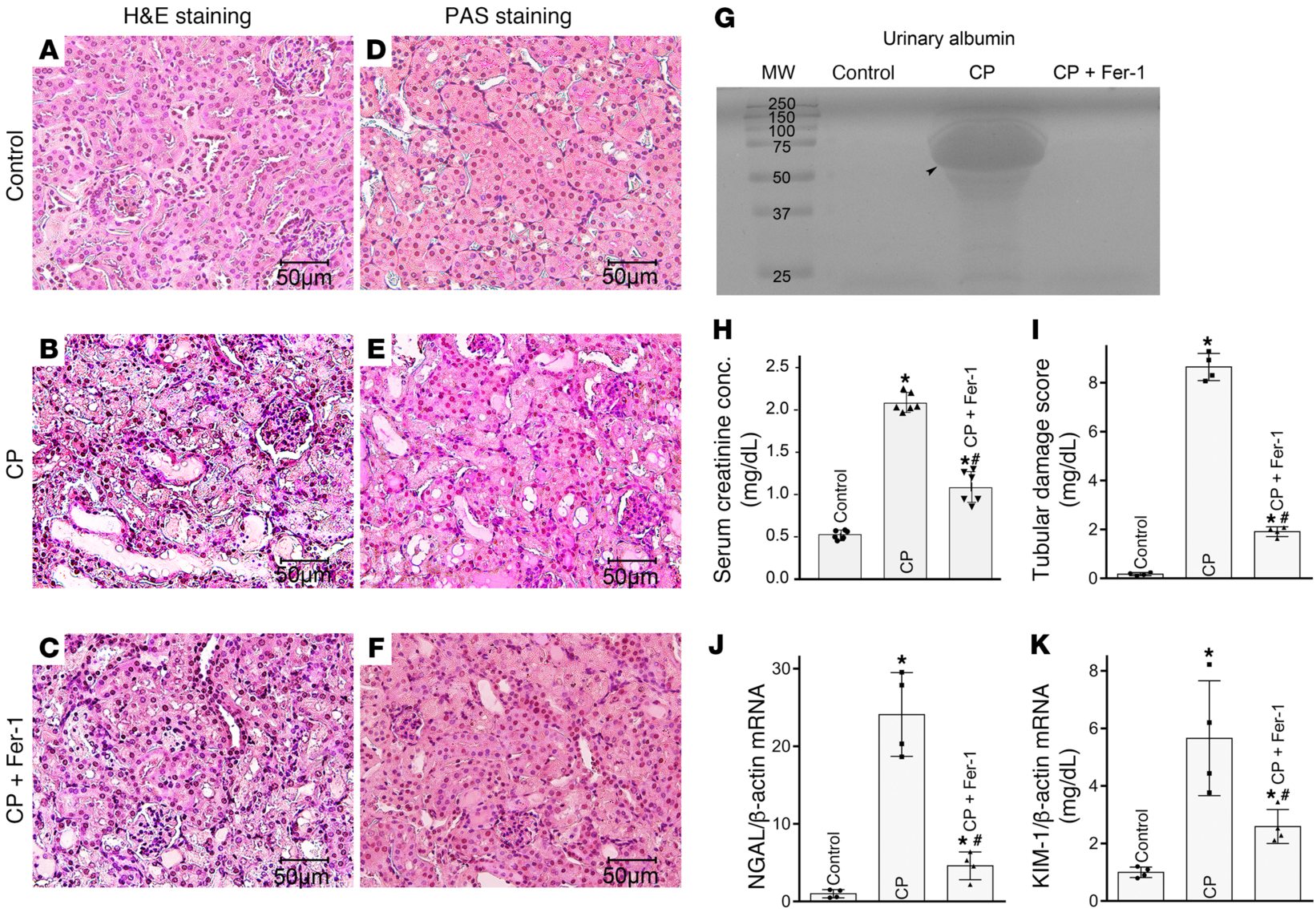

Figure 3. Ferroptosis inhibition attenuates cisplatin-induced AKI. Cisplatin treatment led to a disruption of tubular epithelia, loss of brush borders, and cast formation, which were alleviated by the administration of Fer-1 (A-C). In addition, PAS staining revealed sloughing off of the epithelia and shedding of PAS-positive material in the tubular lumina following cisplatin treatment (E vs. D). These changes were attenuated by the prior treatment of Fer-1 (F vs. E). As assessed by SDS-PACE, cisplatin treatment increased urinary albumin excretion, but not in mice pretreated with Fer-1 (C). Similarly, Fer-1 treatment attenuated cisplatin-induced elevation of serum creatinine levels $(\mathbf{H})\left(n=6 ;{ }^{*} P<0.05\right.$ compared with the control group, ${ }^{\#} P<0.05$ compared with the $\mathrm{CP}$ group, 1-way ANOVA with Dunn's multiple comparisons). Besides, the increase in tubular damage score and mRNA levels of KIM-1 and NGAL induced by cisplatin was also alleviated by the administration of Fer-1 (I-K) $\left(n=4 ;{ }^{*} P<0.05\right.$ compared with the control group, ${ }^{\#} P<0.05$ compared with the CP group, 1-way ANOVA with Dunn's multiple comparisons). Scale bars: $50 \mu \mathrm{m}$.

in cisplatin-treated mice. The excretion was minimal in the control mice and mice treated with Fer-1, suggesting that cisplatin led to a compromise in the tubular absorptive capacity with excretion of urinary protein, and it was alleviated by the inhibition of ferroptosis (Figure 3G). Notably, the serum creatinine levels, tubular damage scores, and mRNA levels of NGAL and KIM-1 (AKI markers) increased following cisplatin treatment. Interestingly, these changes were attenuated by the administration of Fer-1 (Figure 3, $\mathrm{H}-\mathrm{K})$, suggesting that cisplatin induces notable renal functional deterioration by adversely affecting the tubular compartment, and they were alleviated by the inhibition of ferroptosis. Moreover, the perturbation in ferroptosis metabolic sensors, induced by cisplatin, was partially reversed by Fer-1 treatment (Supplemental Figure 1; supplemental material available online with this article; https://doi.org/10.1172/JCI129903DS1). Fer-1 treatment 2 hours prior to the administration of cisplatin also alleviated the tubular injury (Supplemental Figure 2).

Cisplatin promotes ROS generation and accentuates MIOX overexpression, leading to lipid hydroperoxidation in cisplatin-treated HK-2 cells. Mitochondrial ROS generation was assessed by dihydroethidium (DHE) staining. Cisplatin treatment for approxi- mately 20 hours led to a considerable increase in DHE staining (red fluorescence) in HK-2 cells (Figure 4, B vs. A, and D). To confirm the specificity of DHE staining, the mitochondrial ROS scavenger MitoQ was used. The cisplatin-induced increase in DHE staining was partially quenched by MitoQ treatment (Figure 4, C vs. B, and D). MIOX expression increased in HK-2 cells after 4 hours of cisplatin treatment, which was attenuated by MitoQ treatment (Figure 4F, left panel, and Supplemental Figure 3). However, no obvious MIOX upregulation was observed after 20 hours of cisplatin treatment (Figure $4 \mathrm{~F}$, right panel); this may possibly be due to the overwhelming cellular damage that occurred during this prolonged period. To further elucidate the relevance of MIOX in ferroptosis in vitro, the MIOX-overexpressing HK-2 cell line, generated in our laboratory, and MIOX siRNA were used. MIOX overexpression and gene disruption were confirmed by immunoblotting procedures (Figure $4 \mathrm{~F}$, right panel, third, fourth, and sixth lanes). The status of 4-hydroxynonenal (4-HNE), the end product of lipid hydroperoxidation, was evaluated by immunofluorescence microscopy. Normally, there was a minimal background immunofluorescence related to 4-HNE in control untreated cells (Figure 4, E and G). The MIOX-overexpressing 
DHE staining
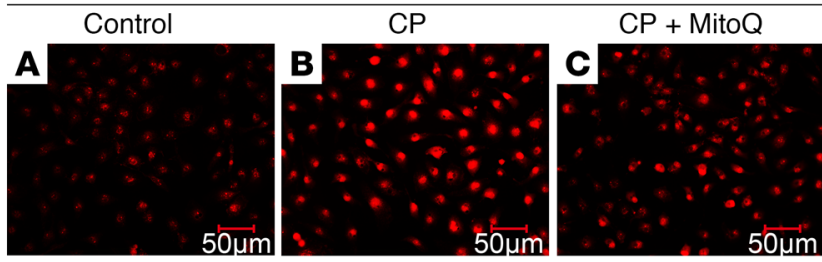

D

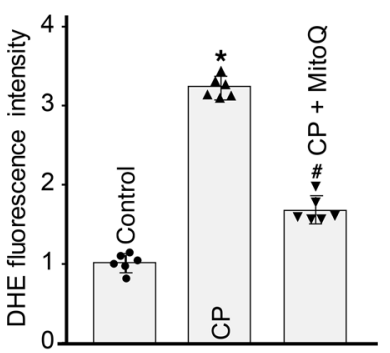

E

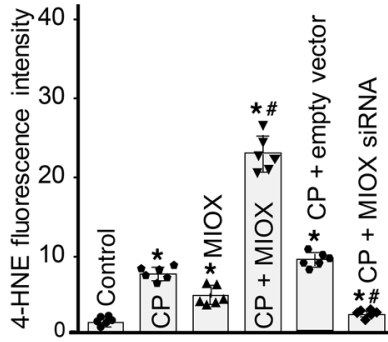

$\mathbf{F}$

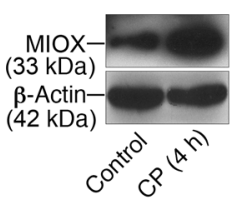

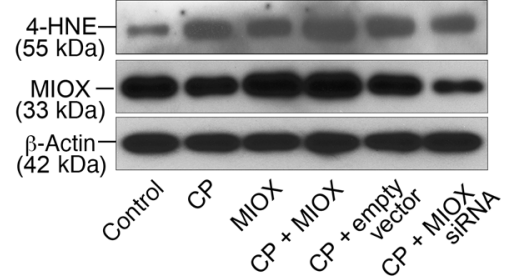

4-HNE staining
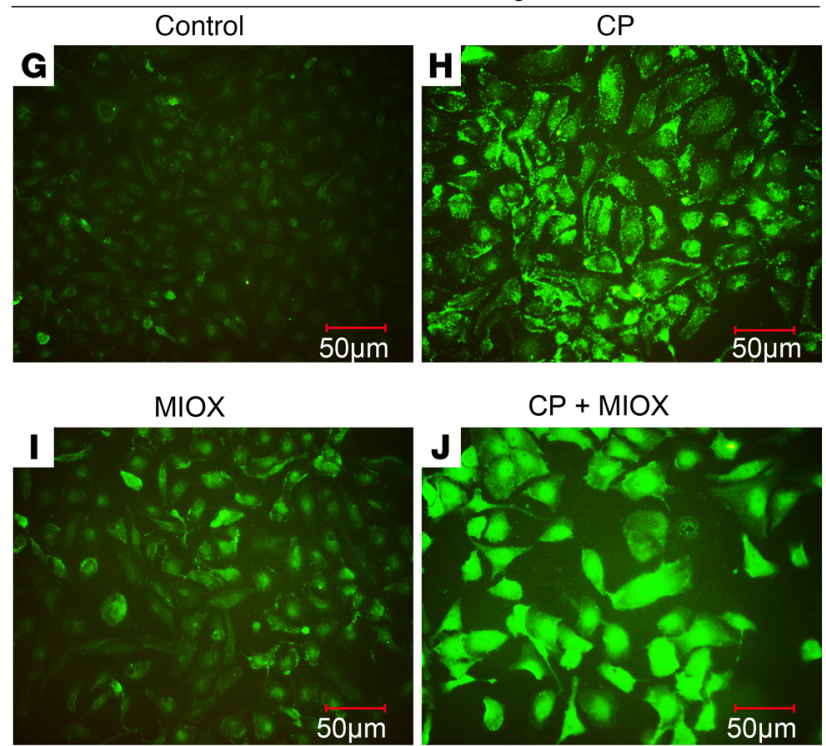

$\mathrm{CP}+$ empty vector

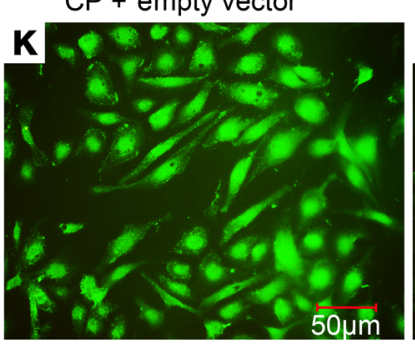

CP + MIOX siRNA

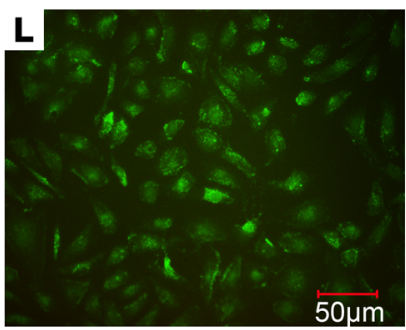

Figure 4. Cisplatin leads to excessive mitochondrial ROS generation and MIOX overexpression, which in turn accentuates cisplatin-induced lipid hydroperoxidation in HK-2 cells. Cisplatin treatment increased DHE staining, indicative of mitochondrial ROS, in HK-2 cells, and it was partially reduced by MitoQ treatment (A-D) ( $n=6$; ${ }^{*} P<0.05$ compared with the control group, ${ }^{\#} P<0.05$ compared with the CP group, 1-way ANOVA with Dunn's multiple comparisons). Immunoblotting studies revealed increased expression of MIOX following 4 hours of cisplatin treatment (F, left). No obvious MIOX upregulation was observed after 20 hours of cisplatin treatment (F, right). Besides, the status of MIOX overexpression and gene disruption in HK-2 cells was confirmed by immunoblotting studies (F, right; third, fourth, and sixth lanes). Fluorescence microscopy revealed that cisplatin treatment for 20 hours led to increased 4-HNE staining, indicative of lipid hydroperoxidation, in HK-2 cells $(\mathbf{E}, \mathbf{G}$, and $\mathbf{H})\left(n=6 ;{ }^{*} P<0.05\right.$ compared with the control group, ${ }^{*} P<0.05$ compared with the CP group, 1-way ANOVA with Dunn's multiple comparisons). The hydroperoxidation was accentuated by the overexpression of MIOX while attenuated by MIOX gene disruption (E and I-L). Similar changes in 4-HNE levels in vitro were observed by immunoblotting analyses (F, right). Scale bars: $50 \mu \mathrm{m}$.

cells or cells treated with cisplatin showed a moderate degree of fluorescence, while cisplatin-treated MIOX-overexpressing cells yielded the maximal degree of fluorescence (Figure $4, \mathrm{E}$ and $\mathrm{H}-\mathrm{J}$ ). Interestingly, MIOX siRNA treatment markedly attenuated the fluorescence in comparison with the control (Figure 4, E and L vs. $\mathrm{K})$. The changes in 4-HNE levels in vitro were further confirmed by immunoblotting analysis (Figure 4F, right panel).

Overexpression of MIOX promotes, while its gene disruption alleviates, cisplatin-induced cell death and RSL3-induced ferroptosis in HK-2 cells. Severe DNA damage, as shown by TUNEL staining, was observed in cisplatin-treated HK-2 cells (Figure 5, $\mathrm{B}$ vs. A). The DNA damage was further accentuated in cisplatintreated MIOX-overexpressing cells (Figure 5, D vs. B and C), while it was remarkably reduced by MIOX siRNA in cisplatintreated cells (Figure 5, F vs. E). Both control HK-2 cells and MIOX-overexpressing cells had a cobblestone epithelial morphology in culture at 48 hours (Figure 5, G and I). Cisplatin treatment led to morphological shrinkage of the cells, and the maximal contraction was seen in cisplatin-treated MIOX-over- expressing cells (Figure 5, J vs. H). Interestingly, transfection with MIOX siRNA partially restored the morphology of HK-2 cells treated with cisplatin (Figure 5, L vs. K). MTT assay revealed that cisplatin-induced $\mathrm{HK}-2$ cell death was alleviated by MIOX siRNA treatment, while it was accentuated by MIOX overexpression (Figure 5, M and N). To further elucidate the role of MIOX in ferroptosis, RSL3, a GPX4 inhibitor, was used to induce ferroptosis in HK-2 cells. MTT studies showed that RSL3 led to massive cell death after 6 hours via the process of ferroptosis (Figure 5, O and P). This ferroptosis-specific cell death, induced by RSL3, was exacerbated by MIOX overexpression while attenuated by MIOX gene disruption (Figure 5, O and P).

Overexpression of MIOX promotes, whereas its gene disruption inhibits, ferritinophagy in cisplatin-treated HK-2 cells. Ferritinophagy, a newly discovered process of selective autophagy, is the autophagic degradation of ferritin in the lysosomes, which is guided by a specific cargo receptor, nuclear receptor coactivator 4 (NCOA4) (16). It is conceivable that intracellular ferritin and NCOA4 are depleted simultaneously by lysosomal degradation in 
TUNEL staining

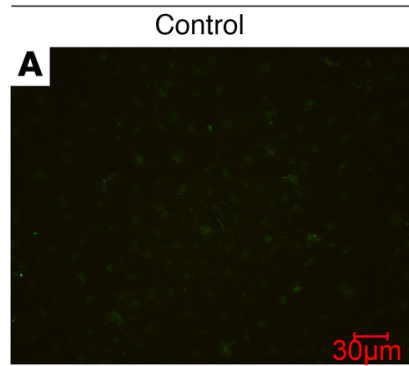

MIOX

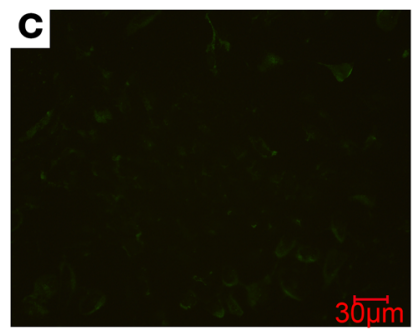

$\mathrm{CP}+$ empty vector

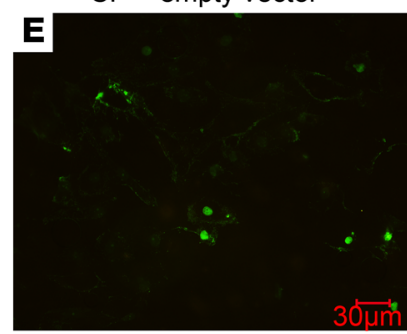

M

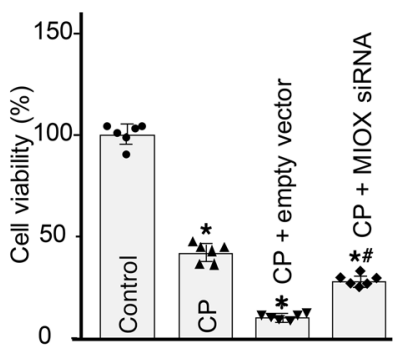

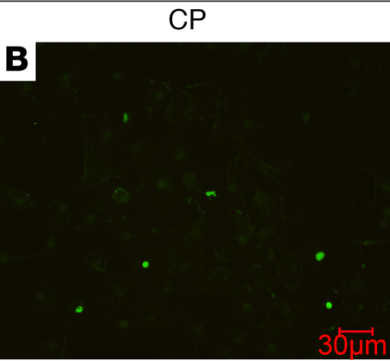

$\mathrm{CP}+\mathrm{MIOX}$

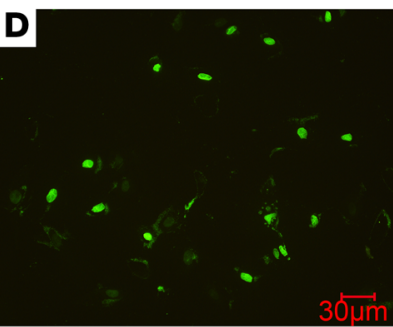

CP + MIOX siRNA

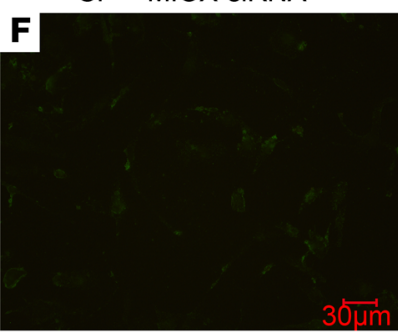

$\mathbf{N}$

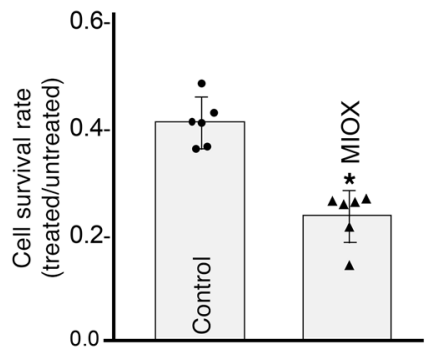

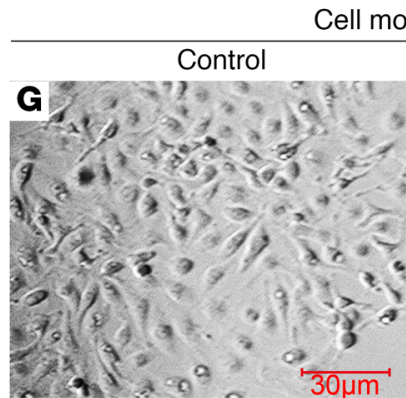

MIOX

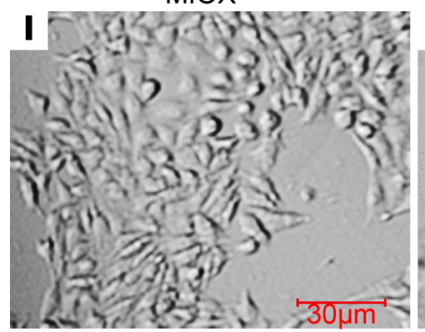

$\mathrm{CP}+$ empty vector

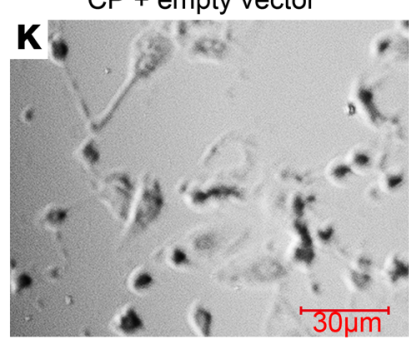

0

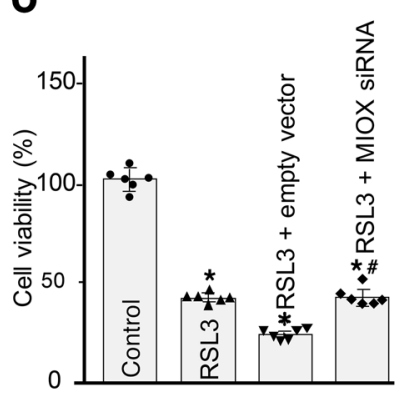

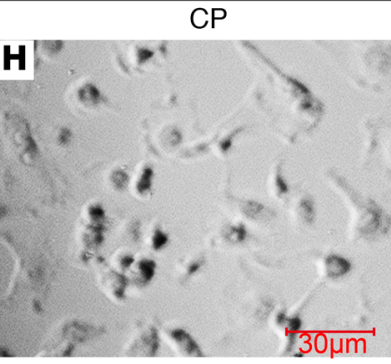

$\mathrm{CP}+\mathrm{MIOX}$

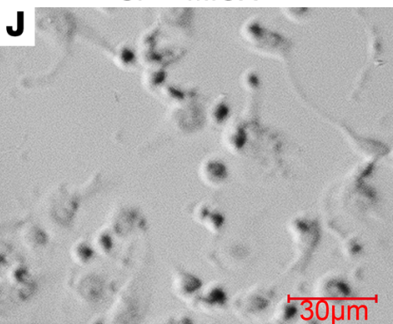

CP + MIOX SiRNA

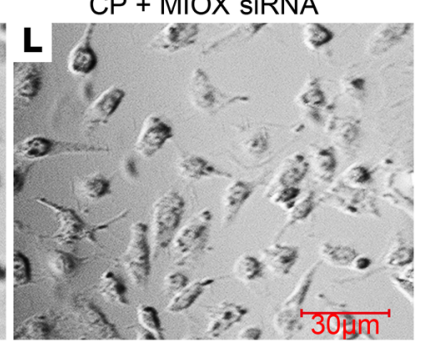

$\mathbf{P}$

Figure 5. Overexpression of MIOX exacerbates cell death, while its gene disruption inhibits it, in cisplatin-treated HK-2 cells. TUNEL staining revealed severe DNA damage in cisplatin-treated HK-2 cells, which was further promoted by MIOX overexpression, and alleviated by MIOX gene disruption (A-F). Cisplatin treatment led to shrinkage of HK-2 cells after 48 hours, and the maximal contraction was seen in MIOX-overexpressing cells, as compared with the controls (G-J). MIOX siRNA treatment partially reversed these changes (L vs. K). As indicated by MTT assay, cisplatin-induced cell death was alleviated by MIOX siRNA treatment; however, it was accentuated by MIOX overexpression ( $\mathbf{M}$ and $\mathbf{N})\left(n=6 ;{ }^{*} P<0.05\right.$ compared with the control group, ${ }^{\#} P<0.05$ compared with the CP + empty vector group, 1-way ANOVA with Dunn's multiple comparisons for $\mathbf{M}$ and 2-tailed Student's $t$ test for N). MTT experiments also revealed that RSL3 (ferroptosis inducer) caused massive ferroptosis-specific cell death in HK-2 cells, which was exacerbated by overexpression of MIOX and attenuated by its gene disruption ( $\mathbf{O}$ and $\mathbf{P})\left(n=6 ;{ }^{*} P<0.05\right.$ compared with the control group, ${ }^{*} P<0.05$ compared with the RSL3 + empty vector group, 1-way ANOVA with Dunn's multiple comparisons for $\mathbf{O}$ and 2-tailed Student's $t$ test for $\mathbf{P}$ ). Scale bars: $30 \mu \mathrm{m}$.

the state of ferritinophagy. In view of this contention, the status of FTH1 and NCOA4 degradation was investigated in the context of ferritinophagy in cisplatin-treated HK-2 cells. Immunofluorescence microscopy revealed intense fluorescence related to the expression of NCOA4 in control HK-2 cells (Figure 6A). A notable decrease in fluorescence was observed in cisplatin-treated HK-2 cells, which was seen maximally in cisplatin-treated MIOX-overexpressing cells, as compared with the controls (Figure 6, A-D). Interestingly, NCOA4-related fluorescence was partially restored following the transfection of cisplatin-treated cells with MIOX siRNA (Figure 6, F vs. E). Similarly, FTH1, the heavy chain of ferritin, underwent substantial downregulation in cisplatintreated HK-2 cells (Figure 6, H vs. G). Further remarkable depletion of FTH1 was observed in cisplatin-treated MIOX-overexpressing cells, whereas MIOX siRNA treatment restored expression quite similar to that in control cells (Figure 6, I-L). Notably, the decrease of FTH1 in cisplatin-treated MIOX-overexpressing cells was partially reversed by the administration of NCOA4 

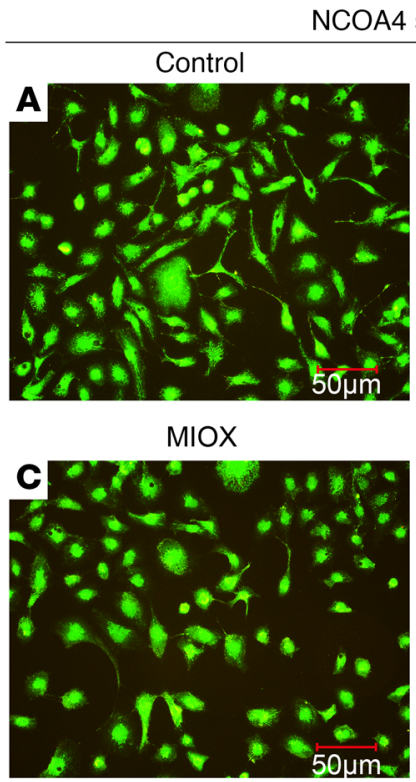

$$
\mathrm{CP}+\text { empty vector }
$$

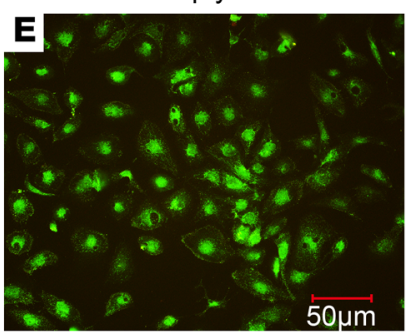

M

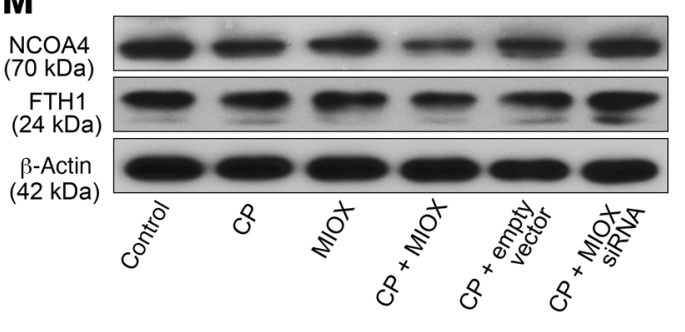

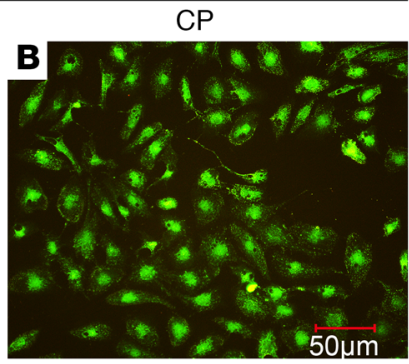

$\mathrm{CP}+\mathrm{MIOX}$

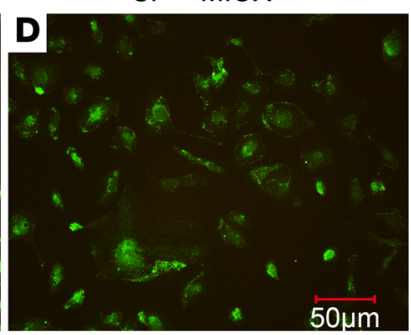

CP + MIOX SiRNA

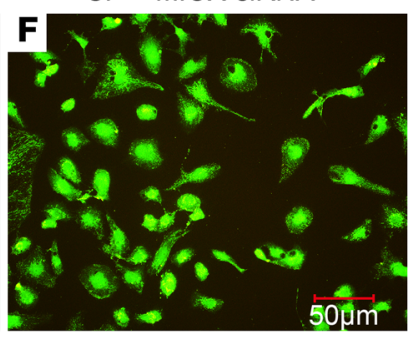

FTH1 staining

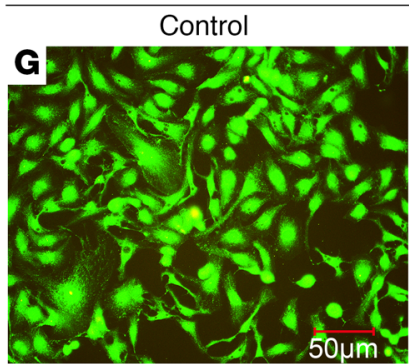

MIOX
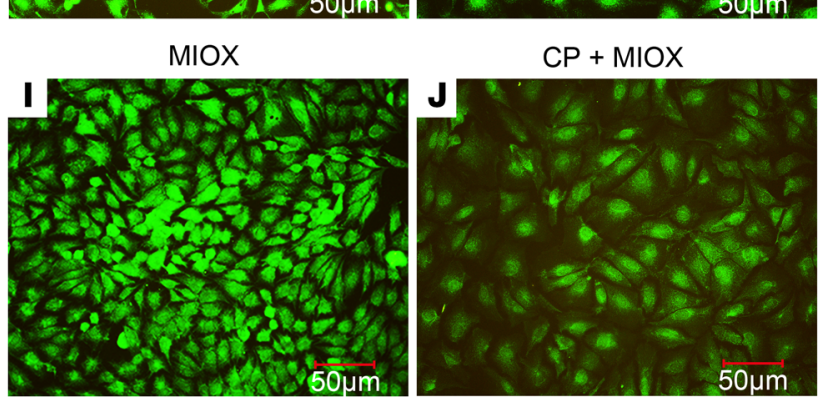

$\mathrm{CP}+$ empty vector
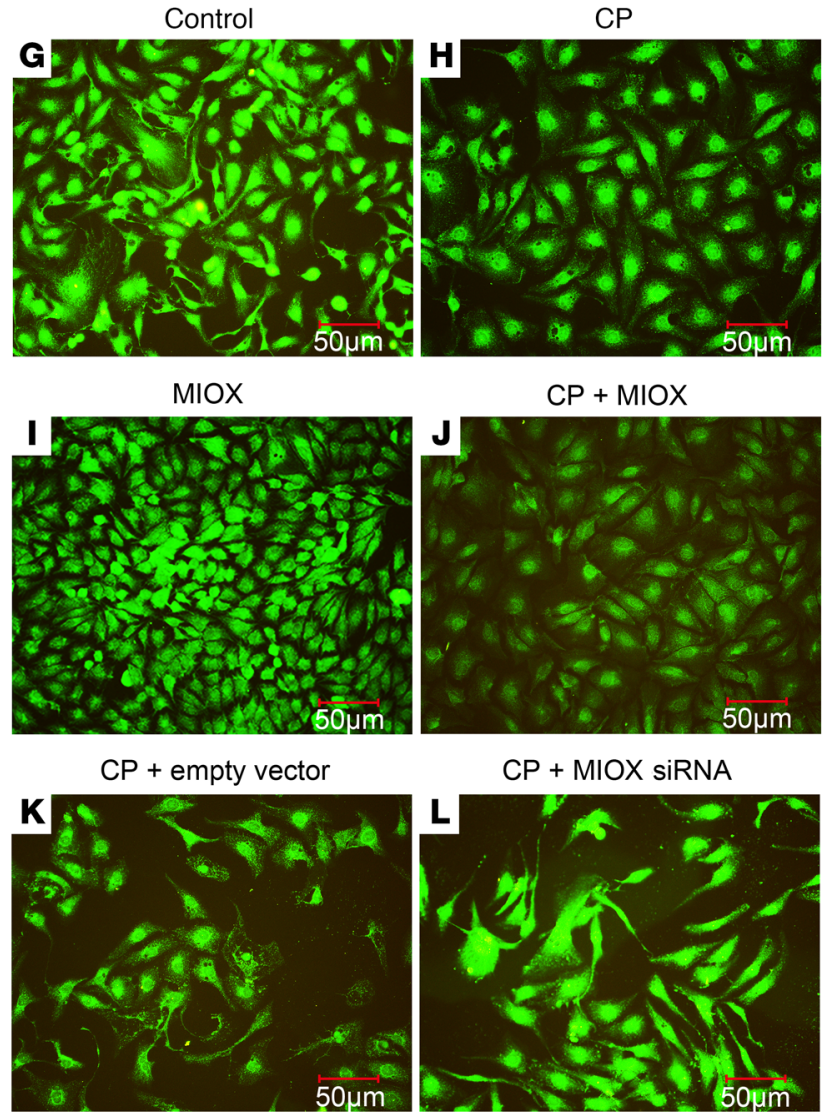

$\mathbf{N}$

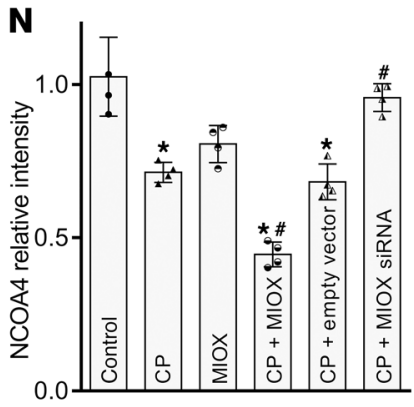

o

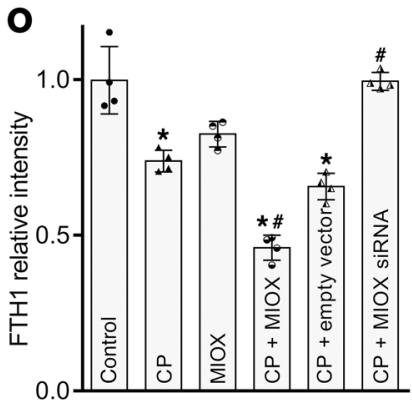

Figure 6. Knockdown of MIOX inhibits, while its overexpression accelerates, ferritinophagy in cisplatin-treated HK-2 cells. Immunofluorescence microscopy revealed a decreased fluorescence related to the expression of NCOA4 in cisplatin-treated HK-2 cells (B vs. A). The immunofluorescence intensity was maximally reduced in cisplatin-treated MIOX-overexpressing cells, and it was partially restored following MIOX siRNA treatment (C-F). Similarly, there was a substantial downregulation of FTH1 in cisplatin-treated HK-2 cells, and a further decrease in FTH1-related immunofluorescence was observed in cisplatin-treated MIOX-overexpressing cells, whereas MIOX siRNA treatment prevented its downregulation (G-L). The changes in NCOA4 and FTH1 expression levels were confirmed by immunoblotting studies $(\mathbf{M}-\mathbf{0})\left(n=4 ;{ }^{*} P<0.05\right.$ compared with the control group, ${ }^{P} P<0.05$ compared with the CP group, 1-way ANOVA with Dunn's multiple comparisons). Scale bars: $50 \mu \mathrm{m}$.

siRNA (Supplemental Figure 4). The results of immunoblotting studies were similar to the observations made by immunofluorescence microscopy, and they revealed that MIOX heavily influenced the degradation of FTH1 and NCOA4 in HK-2 cells following cisplatin treatment (Figure 6, M-O). Interestingly, the depletion of FTH1 was also observed in the early stage (4 hours) of cisplatin treatment, which could be alleviated by MitoQ treatment (Supplemental Figure 3).

MIOX gene disruption attenuates lysosomal ferritin uptake and intracellular free iron accumulation, while MIOX overexpression enhances these processes, in cisplatin-treated HK-2 cells. Free iron is required for lipid hydroperoxidation and execution of ferroptosis, but most of the intracellular iron remains bound to ferritin (9). In the state of ferritinophagy, ferritin is phagocytosed by lysosomes and then undergoes enzymatic degradation, leading to excessive generation of free iron. In view of these interrelated dynamics, lysosome and ferritin were costained to elucidate the lysosomal uptake of ferritin in HK-2 cells. Lysosomal-associated membrane protein 1 (LAMP1), a commonly used lysosomal marker, was used to stain the lysosomes. Immunofluorescence 
FTH1, LAMP1, and DAPI staining

Control
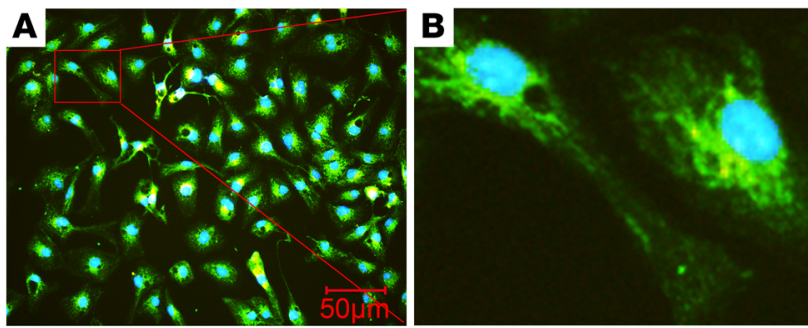

MIOX
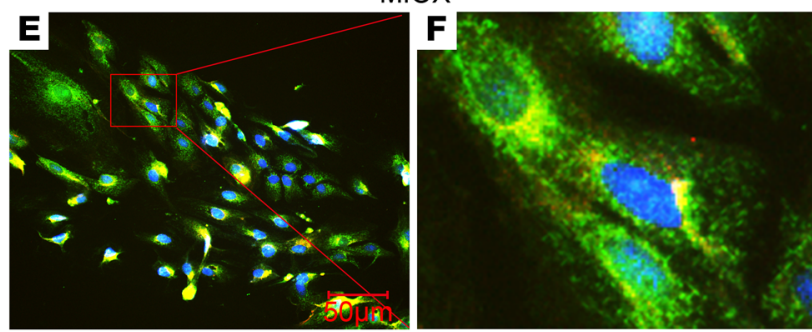

$\mathrm{CP}+$ empty vector
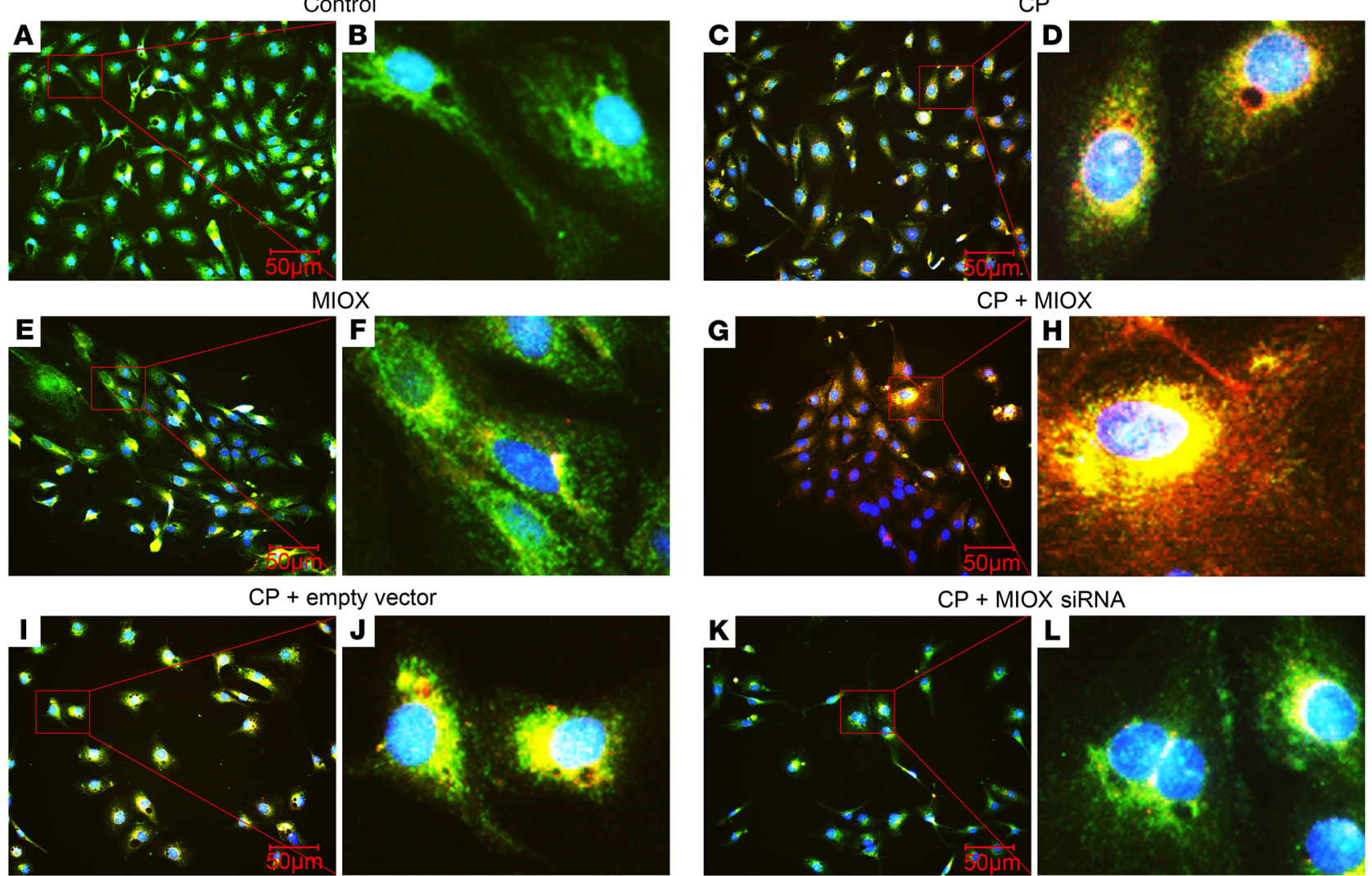

$\mathrm{CP}+\mathrm{MIOX}$
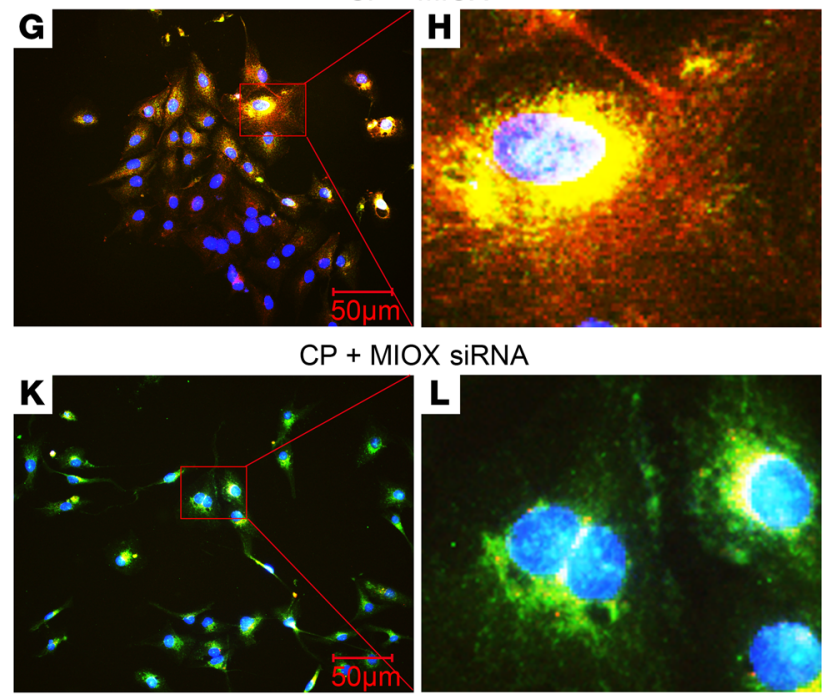

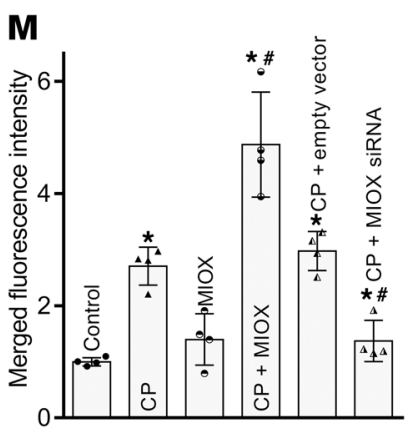

$\mathbf{N}$

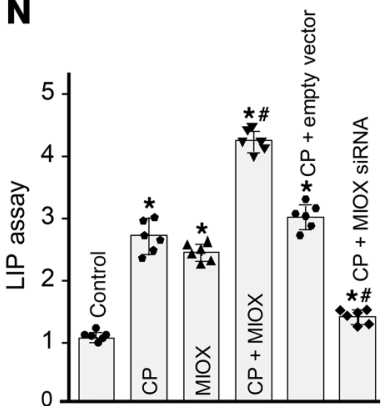

Figure 7. Overexpression of MIOX accentuates, whereas its gene disruption attenuates, ferritin uptake by the lysosomes and accumulation of free iron after cisplatin treatment. Normally, ferritin (FTH1, green) was seen localized primarily in the cytoplasm and in small amounts in the lysosome (LAMP1, red) in untreated HK-2 cells ( $\mathbf{A}$ and $\mathbf{B}$ ). Immunofluorescence microscopy revealed considerable translocation of FTH1 into the lysosomal compartment in cisplatin-treated HK-2 cells (C, $\mathbf{D}$, and $\mathbf{M})\left(n=4 ;{ }^{*} P<0.05\right.$ compared with the control group, ${ }^{\#} P<0.05$ compared with the CP group, 1 -way ANOVA with Dunn's multiple comparisons). The translocation was tremendously enhanced in cisplatin-treated MIOX-overexpressing cells, while remarkably disrupted by the transfection of MIOX siRNA (E-M). To measure intracellular free iron levels, labile iron pool (LIP) assays were performed. The results indicated a marked increase of intracellular free iron concentration in HK-2 cells after cisplatin treatment $(\mathbf{N})\left(n=4 ;{ }^{*} P<0.05\right.$ compared with the control group, ${ }^{\#} P<$ 0.05 compared with the CP group, 1-way ANOVA with Dunn's multiple comparisons). The concentration of free iron was seen further increased in cisplatin-treated MIOX-overexpressing cells, and it was attenuated by MIOX gene disruption (N). Scale bars: $50 \mu \mathrm{m}$.

microscopy revealed considerable colocalization of FTH1 (green) and LAMP1 (red) in cisplatin-treated HK-2 cells, indicating the translocation of ferritin into the lysosomes (Figure 7, C and $\mathrm{D}$ vs. A and $\mathrm{B}$, and $\mathrm{M}$ ). The translocation was tremendously enhanced in cisplatin-treated MIOX-overexpressing cells, as can be readily visualized in Figure 7, G, H, and M. Transfection of MIOX siRNA into cisplatin-treated cells remarkably disrupted the codistribution (Figure 7, $\mathrm{K}$ and $\mathrm{L}$ vs. I and J, and M). To measure intracellular free iron levels, labile iron pool assay was per- formed. The results indicated a marked increase of intracellular free iron concentration in HK-2 cells after cisplatin treatment, which was accentuated in cisplatin-treated MIOX-overexpressing cells, and attenuated by MIOX gene disruption (Figure $7 \mathrm{~N}$ ).

MIOX overexpression modulates lysosomal permeability, GPX4 activity, and reduced glutathione and NADPH levels in HK-2 cells following cisplatin treatment. Since ferroptosis is akin to autophagic cell death, it is conceivable that the lysosomal permeability of HK-2 cells increases during the ferroptotic process (17). In view of 


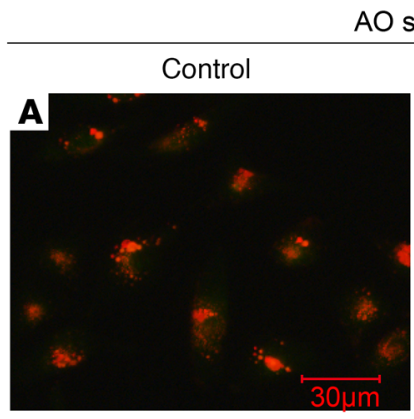

MIOX

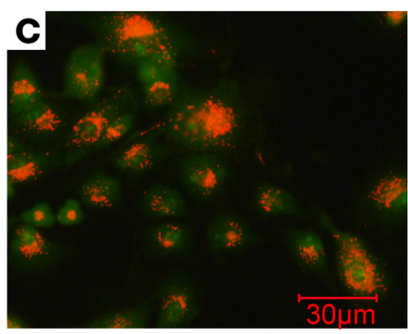

$\mathrm{CP}+$ empty vector

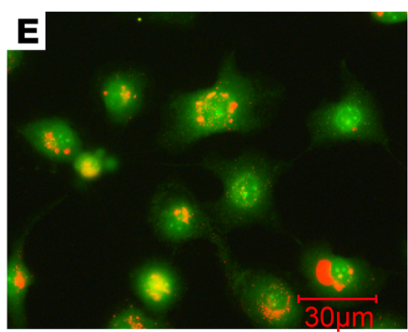

M

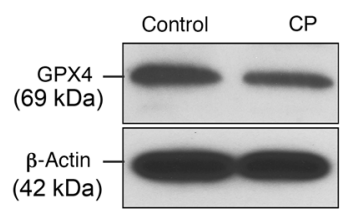

$\mathrm{CP}$

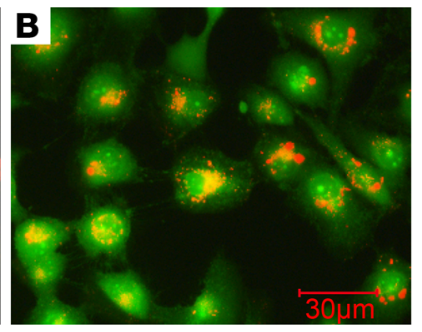

$\mathrm{CP}+\mathrm{MIOX}$

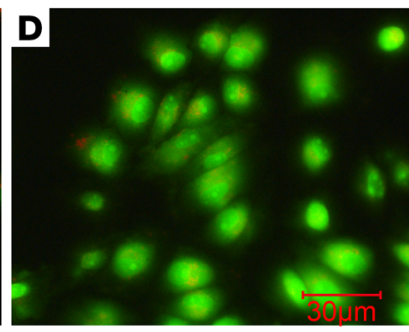

CP + MIOX SIRNA

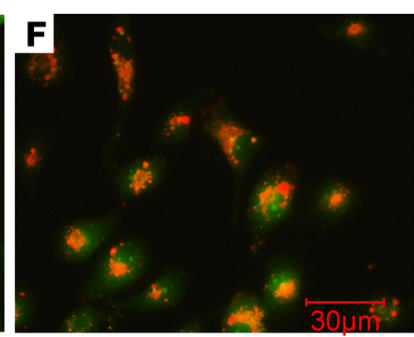

MBB staining

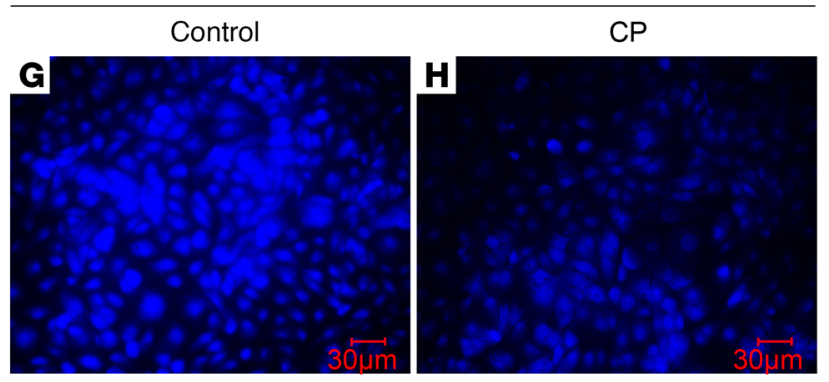

MIOX
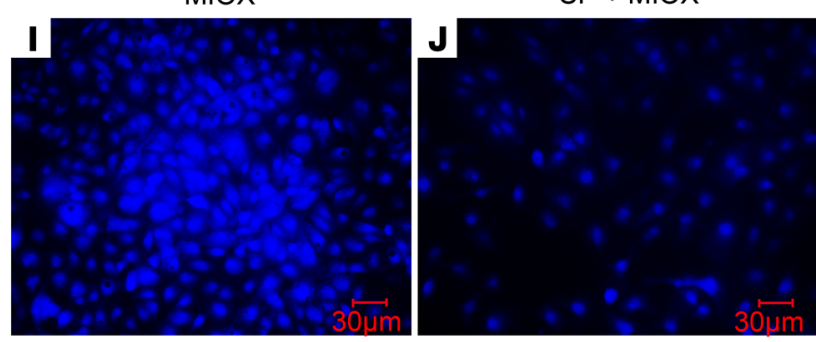

$\mathrm{CP}+$ empty vector
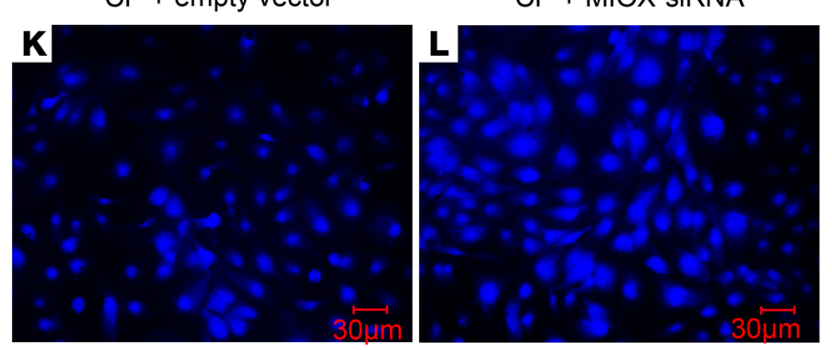

$\mathbf{N}$

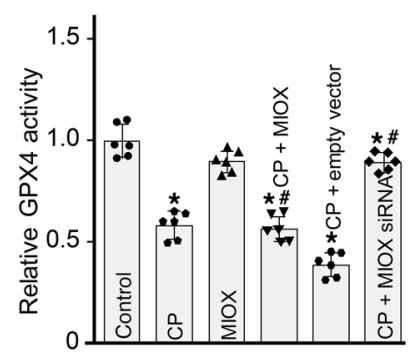

0

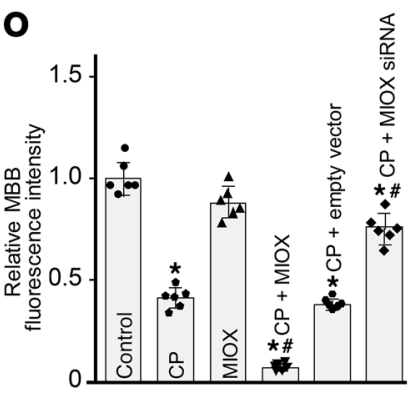

\section{P}

Figure 8. MIOX overexpression promotes lysosomal permeability and decreases GSH concentration, GPX4 activity, and NADPH levels in cisplatintreated HK-2 cells. Lysosomal permeability was investigated by AO staining. AO-associated green fluorescence in the cytoplasm increased, while red fluorescence in the lysosome decreased, suggesting increased lysosomal permeability of HK-2 cells following cisplatin treatment (B vs. A). The changes in fluorescence were accentuated in cisplatin-treated MIOX-overexpressing cells but attenuated in cisplatin-treated cells transfected with MIOX siRNA (C-F). The expression of GPX4, a key enzyme for ferroptosis inhibition, decreased after cisplatin treatment (M). A substantial decline in GPX4 activity was also observed in both HK-2 cells and MIOX-overexpressing cells, and this decrease was negated by the concomitant transfection with MIOX siRNA (N) ( $n=6$; ${ }^{*} P<0.05$ compared with the control group, ${ }^{\#} P<0.05$ compared with the CP group, 1 -way ANOVA with Dunn's multiple comparisons). The status of intracellular GSH levels was assessed by monobromobimane (MBB) staining. Blue fluorescence was decreased after cisplatin treatment, and further decreased in cisplatin-treated MIOX-overexpressing cells, while partially restored by MIOX siRNA transfection (G-L and $\mathbf{0})\left(n=6\right.$; ${ }^{*} P<0.05$ compared with the control group, ${ }^{~} P<0.05$ compared with the CP group, 1-way ANOVA with Dunn's multiple comparisons). The NADPH levels were also found to be low in MIOX-overexpressing cells and cisplatin-treated cells $\mathbf{( P )}$. There was further depletion of NADPH in cisplatin-treated MIOX-overexpressing cells, which was blocked by MIOX gene disruption (P) ( $n=6$; ${ }^{*} P<0.05$ compared with the control group, ${ }^{*} P<0.05$ compared with the CP group, 1-way ANOVA with Dunn's multiple comparisons). Scale bars: $30 \mu \mathrm{m}$.

this notion, the status of lysosomal permeability was investigated by acridine orange (AO) dye staining. In untreated cells, most of the dye accumulates inside the lysosomes to generate red fluorescence, and a small portion of it remains in the cytoplasm to yield green fluorescence. When lysosomal permeability increases, AO leaks from lysosomes into cytoplasm, and the cells yield waxing of green fluorescence and waning of red fluorescence. In this study, we noted that $\mathrm{AO}$-associated green fluorescence increased and red fluorescence decreased in cisplatin-treated HK-2 cells (Figure 8, B vs. A). These changes in fluorescence were accentuated in cispla- 
(PAS)
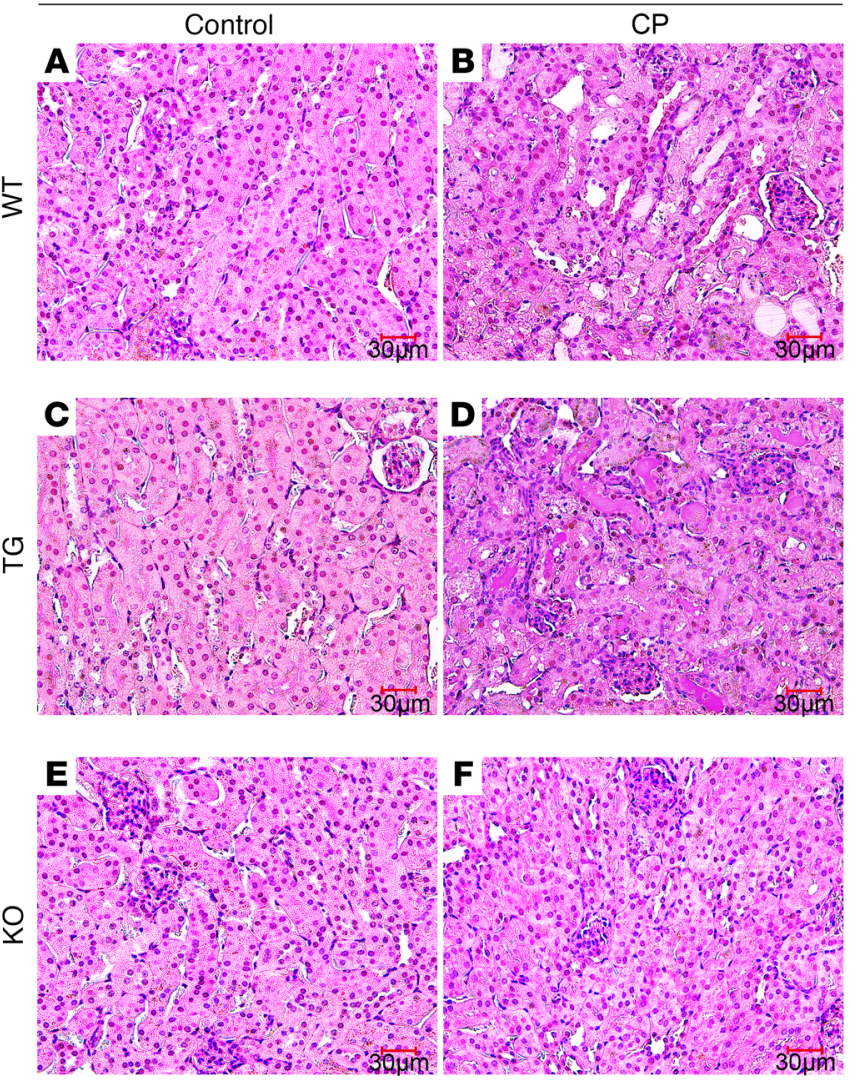

$\mathbf{M}$
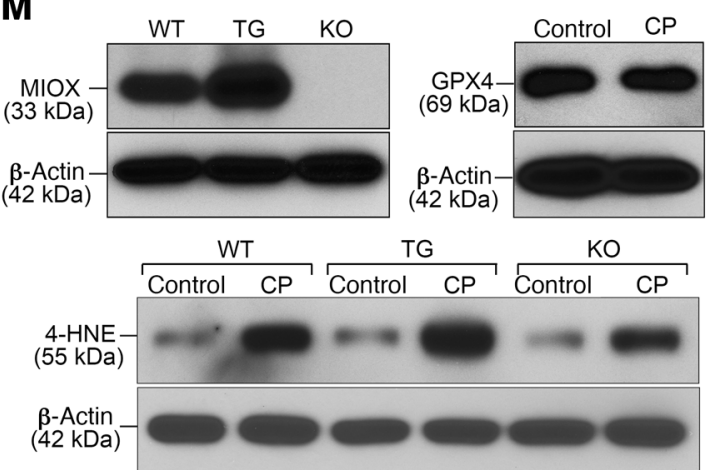

$\mathbf{N}$

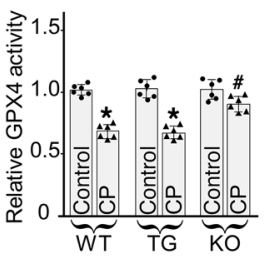

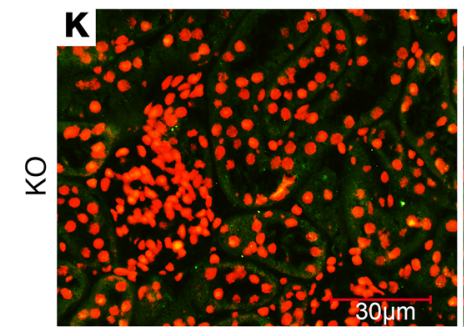

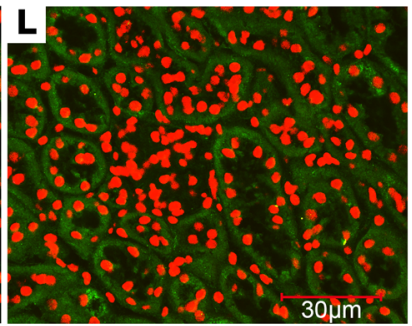

0

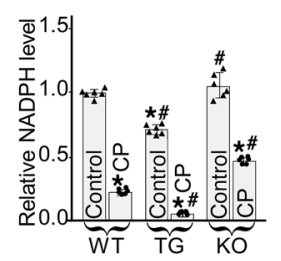

(4-HNE)
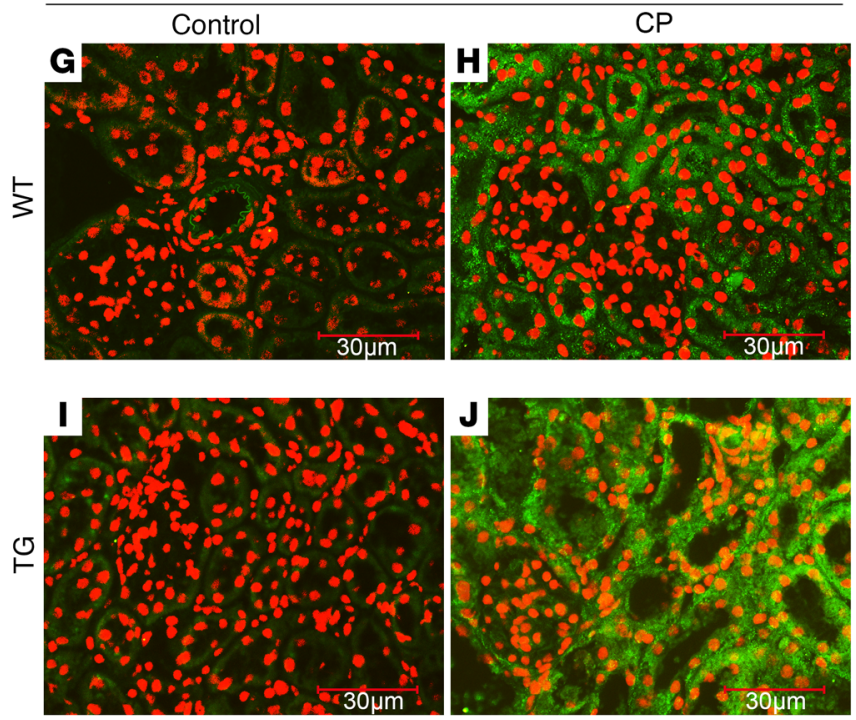

$\mathbf{P}$

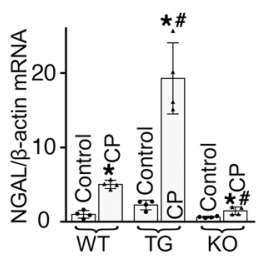

Figure 9. Overexpression of MIOX exacerbates, while its gene disruption alleviates, renal tubular injury, lipid hydroperoxidation, and decline in GPX4 activity and NADPH levels in cisplatin-induced AKI. The expression profile of MIOX in WT, MIOX-Tg, and MIOX-KO mice was demonstrated by immunoblotting studies (M). Cisplatin treatment led to severe renal tubular injury in WT mice, which was accentuated in MIOX-Tg mice but attenuated in MIOX-KO mice (A-F). Similarly, NGAL mRNA levels increased in cisplatin-treated WT mice and MIOX-Tg mice, and a minimal increase was observed in cisplatin-treated MIOX-KO mice (P) $\left(n=4 ;{ }^{*} P<0.05\right.$ compared with the WT control group, ${ }^{\#} P<0.05$ compared with the WT CP group, 1 -way ANOVA with Dunn's multiple comparisons). 4-HNE expression levels were used to assess the status of lipid hydroperoxidation. Immunofluorescence and immunoblotting studies revealed an increase in 4-HNE levels in cisplatin-treated WT mice, which was maximal in cisplatin-treated MIOX-Tg mice but minimal in cisplatin-treated MIOX-KO mice (G-M). Besides, a decline in GPX4 activity was observed in cisplatin-treated WT mice and MIOX-Tg mice but not in cisplatin-treated MIOX-KO mice (N) $\left(n=6\right.$; ${ }^{*} P<0.05$ compared with the WT control group, ${ }^{*} P<0.05$ compared with the WT CP group, 1-way ANOVA with Dunn's multiple comparisons), although GPX4 expression remained stable after cisplatin treatment (M). The NADPH levels in untreated MIOX-Tg mice were much lower than those in WT mice (0) $\left(n=6\right.$; ${ }^{*} P<0.05$ compared with the WT control group, ${ }^{*} P<0.05$ compared with the WT CP group, 1 -way ANOVA with Dunn's multiple comparisons). Both WT and MIOX-Tg mice had markedly decreased NADPH levels following cisplatin treatment, while a moderate reduction was seen in cisplatin-treated MIOX-KO mice (0). Scale bars: $30 \mu \mathrm{m}$.

tin-treated MIOX-overexpressing cells (Figure 8, D vs. B and C), whereas the changes were minimal in cisplatin-treated cells transfected with MIOX siRNA, as compared with the controls (Figure 8, F vs. A, C, and E).
GPX4 is a key antioxidant enzyme that can block ferroptosis by eliminating lipid hydroperoxides with reduced glutathione (GSH) in states in which cells experience excessive ferroptotic pressure (18). Conceivably, ferroptosis can be accelerated when 
(NCOA4)
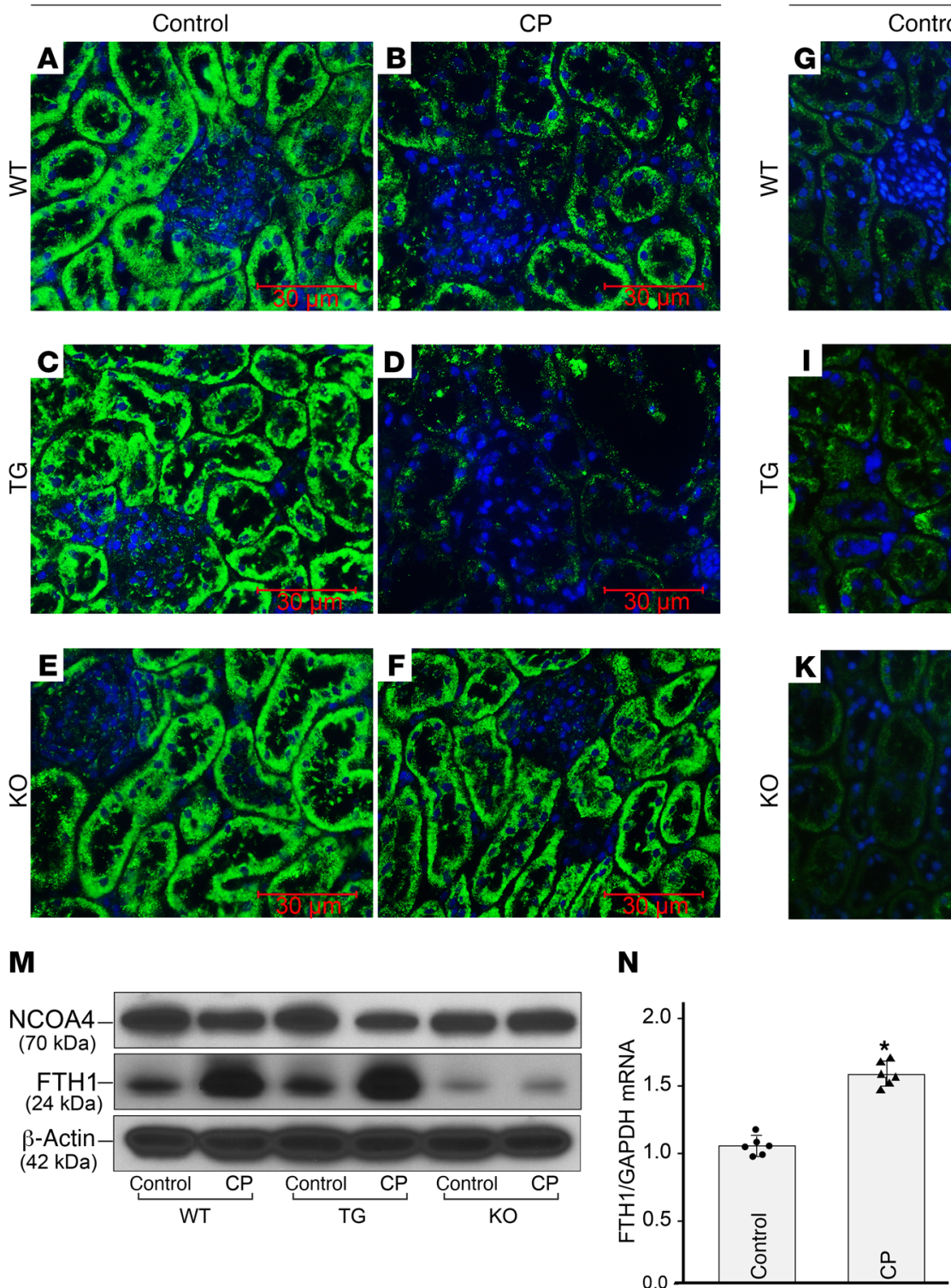

$\mathbf{N}$
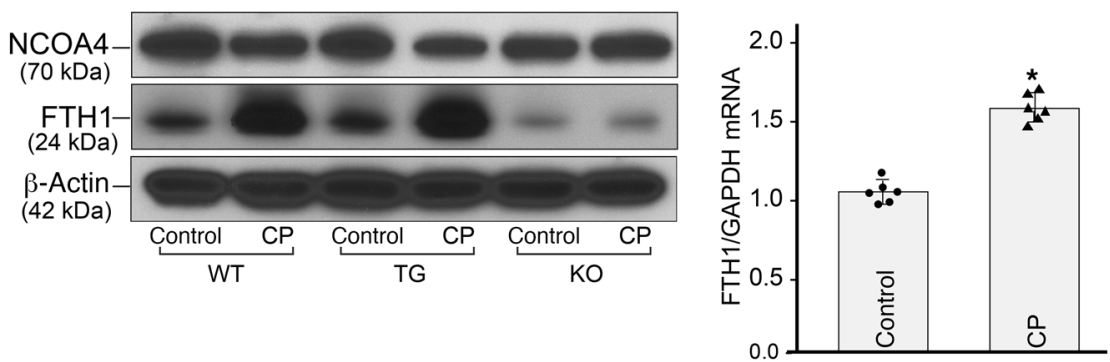

0

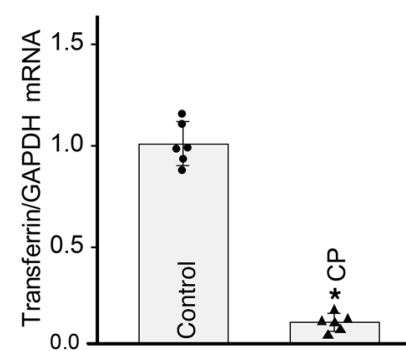

Figure 10. MIOX overexpression promotes ferritinophagy in cisplatin-induced AKI. Immunofluorescence microscopy showed that both ferritin and NCOA4 were mainly expressed in renal tubular epithelia (A-L). The expression of NCOA4 decreased in cisplatin-treated WT mice; however, MIOX-KO mice were unaffected, as indicated by immunofluorescence and immunoblotting studies (B vs. A, F vs. E, and $\mathbf{M}$ ). The maximal decrease in the NCOA4 expression was observed in cisplatin-treated MIOX-Tg mice (D vs. B and C, and $\mathbf{M}$ ). Intriguingly, FTH1 expression increased markedly in cisplatin-treated WT mice (H vs. G, and $\mathbf{M}$ ). This upregulation may be due to the feedback mechanism (21), which was substantiated by reverse transcriptase PCR analyses in the present study. The analyses revealed increased FTH1 and decreased transferrin mRNA levels in cisplatin-treated kidneys ( $\mathbf{N}$ and $\mathbf{0})\left(n=6\right.$; ${ }^{*} P<0.05$ compared with the control group, 2-tailed Student's $t$ test). FTH1 expression in cisplatin-treated MIOX-Tg mice was maximally increased, while no obvious changes were noted in cisplatin-treated MIOX-KO mice (J vs. H and I, L vs. K, and M). Scale bars: $30 \mu \mathrm{m}$.

GPX4 is inhibited or GSH is depleted. In view of this, GPX4 activity and GSH concentration were investigated in HK-2 cells. Immunoblotting analysis revealed that GPX4 expression decreased after cisplatin treatment in HK-2 cells (Figure 8M). A substantial decline in GPX4 activity was also observed in both cisplatin-treated HK-2 cells and cisplatin-treated MIOXoverexpressing cells, and this decrease was not noted in cisplatin-treated cells transfected with MIOX siRNA (Figure 8N). The status of intracellular GSH levels was assessed by monobromobimane (MBB) staining. MBB is taken up by live cells and binds with GSH to generate blue fluorescence. Blue fluorescence was considerably decreased after cisplatin treatment and further decreased by MIOX overexpression, but partially restored by MIOX siRNA transfection (Figure 8, G-L and O). In addition, NADPH levels were measured, since they modulate ferroptosis sensitivity and also aid in eliminating lipid hydroperoxides (9). The levels of NADPH were found to be quite low in MIOX-overexpressing cells (Figure 8P), which may be related to its conversion into $\mathrm{NADP}^{+}$during the myo-inositol catabolic pathway (13, 19). Apparently, acceleration of this pathway by MIOX overexpression in cisplatin-induced injury may have led to the NADPH depletion in HK-2 cells. Understandably, such a depletion of 
NADPH can be blocked by MIOX gene disruption, as observed in this investigation (Figure 8P).

Overexpression of MIOX exacerbates, while its gene disruption alleviates, renal tubular injury, lipid hydroperoxidation, and decline in GPX4 activity and NADPH levels in cisplatin-induced AKI. MIOX-transgenic (MIOX-Tg) mice and MIOX-knockout (MIOX$\mathrm{KO})$ mice were generated as previously reported (8). The expression of MIOX was confirmed by the immunoblotting procedures (Figure 9M). Cisplatin (20 mg/kg) or PBS was administered i.p. to WT, MIOX-Tg, and MIOX-KO mice. PAS staining demonstrated tubular epithelium disruption, loss of brush borders, and cast formation in the tubular lumina in cisplatin-treated WT mouse kidneys (Figure 9, B vs. A). These morphological changes were highly accentuated in cisplatin-treated MIOX-Tg mice (Figure 9, D vs. B and C). Interestingly, no notable pathological changes were observed in cisplatin-treated MIOX-KO mice as compared with the controls (Figure 9, F vs. A, C, and E). Similarly, increased NGAL mRNA levels were observed in cisplatin-treated WT mice and MIOX-Tg mice but not in cisplatin-treated MIOX-KO mice (Figure 9P). Notably, the tubular injury in cisplatin-treated MIOXTg mice was attenuated by prior administration of Fer-1 (Supplemental Figure 5). To determine the extent of lipid hydroperoxidation during cisplatin-induced injury, the status of 4-HNE was assessed by immunofluorescence microscopy. The kidney sections were stained with anti-4-HNE (green) and counterstained with TO-PRO-3 iodide to delineate the nuclei (red). The WT mice treated with cisplatin revealed mild greenish cytoplasmic staining compared with the controls (Figure 9, H vs. G). The 4-HNE staining was notably increased in kidney sections of cisplatin-treated MIOX-Tg mice (Figure 9, J vs. $\mathrm{H}$ and I), while minimal 4-HNE staining was observed in cisplatin-treated MIOX-KO mice as compared with the controls (Figure 9, L vs. G, I, and K). The changes in 4-HNE levels were confirmed with immunoblotting studies (Figure 9M). Then, we measured GPX4 activity to evaluate the ferroptosis termination system in vivo. A decline in GPX4 activity was observed in cisplatin-treated WT mice and MIOX$\mathrm{Tg}$ mice but not in cisplatin-treated MIOX-KO mice (Figure 9N), although GPX4 expression remained stable after cisplatin treatment (Figure 9M). Notably, the NADPH levels in untreated MIOX-Tg mice were much lower than those in WT mice (Figure 90), suggesting that MIOX-Tg mice, to begin with, may be more amenable to the process of ferroptosis. Both WT and MIOX-Tg mice had markedly decreased NADPH levels following cisplatin treatment, while a moderate reduction was seen in cisplatintreated MIOX-KO mice (Figure 9O).

MIOX overexpression promotes ferritinophagy in cisplatininduced AKI. Expression of ferritin and of its cargo receptor NCOA4 was investigated to assess the status of ferritinophagy in vivo. Immunofluorescence microscopy showed that both ferritin and NCOA4 were mainly expressed in renal tubular epithelia (Figure 10, A-L). The immunofluorescence and immunoblotting studies revealed that NCOA4 expression was decreased in cisplatintreated WT mice; however, MIOX-KO mice were unaffected (Figure 10, B vs. A, F vs. E, and M). The maximal decrease in NCOA4 expression was observed in cisplatin-treated MIOX-Tg mice (Figure 10, D vs. B and C, and M). However, changes in FTH1 expression in cisplatin-treated WT mice were opposite to that observed for NCOA4. There was a marked increase in FTH1 expression in cisplatin-treated WT mice, as indicated by immunofluorescence and immunoblotting studies (Figure 10, H vs. G, and M). This upregulation might be induced by the accumulated intracellular free iron in the kidney, which can promote FTH1 transcription via a feedback mechanism while inhibiting transferrin transcription at the same time $(20,21)$. Subsequently, reverse transcriptase PCR analyses were performed to assess the transcriptional status of FTH1 and transferrin in the kidneys. Increased FTH1 and decreased transferrin mRNA levels were noted in cisplatintreated kidneys (Figure 10, $\mathrm{N}$ and $\mathrm{O}$ ), implying that there may be a high concentration of intracellular free iron in tubular epithelia. Notably, in contrast to NCOA4, FTH1 expression in cisplatin-treated MIOX-Tg mice was maximally increased, while no obvious increase was noted in MIOX-KO mice (Figure 10, J vs. $\mathrm{H}$ and $\mathrm{I}, \mathrm{L}$ vs. $\mathrm{K}$, and $\mathrm{M}$ ). Interestingly, MIOX-KO mice had very low basal levels of FTH1 (Figure 10, K-M), and the processes of ferritinophagy and the feedback mechanism were induced at a very early time period ( 2 hours) following the administration of cisplatin (Supplemental Figure 6).

\section{Discussion}

AKI is closely associated with a variety of cell death processes, including apoptosis, necroptosis, and ferroptosis. Most of them have been extensively investigated, except ferroptosis, possibly because of its more recent discovery $(7,22)$. Ferroptosis refers to a subclass of cell death characterized by iron overload and fatal iron-catalyzed lipid damage (23-26). Owing to its caspase- and necrosome-independence, ferroptosis differs from apoptosis and necroptosis $(9,27,28)$. Previous publications have shown that blockade of autophagy leads to a notable alleviation of ferroptosis, suggesting that it is most likely reflective of an autophagic cell death process $(21,29)$. Notably, it has been reported that ferroptosis is essential in AKI induced by ischemia/reperfusion injury (IRI), oxalate, or folic acid $(12,27)$. However, its role in cisplatin-induced AKI has not been investigated, although this is perhaps the most suitable model since the tubular injury can be reproducibly achieved in vivo and in vitro. Interestingly, it has been reported that there is disruption of iron homeostasis in cisplatin-treated mouse kidneys (30). Besides, Linkermann et al. observed that there was no difference in the survival kinetics among WT mice, caspase- $8^{-/-}$mice, and mice deficient in receptor-interacting protein kinase 3 (RIPK3 ${ }^{-/}$; necroptosis) following cisplatin treatment (27). This would suggest that, apart from apoptosis and necroptosis, cisplatin-induced tubular injury may be induced by some other mechanism(s) or process. In this study, we observed that cisplatin-induced nephropathy was substantially relieved by Fer-1 (Figure 3). Our in vitro studies also showed that cisplatin-induced HK-2 cell death was attenuated by Fer-1, deferoxamine, and Z-VAD(OMe)-FMK, but not by Nec-1 (Figure 2, A-K), indicating that ferroptosis and apoptosis, but not necroptosis, played a key role in the cellular injury. Similar results have been reported in cisplatin-induced cancer cell death (31). Our studies also showed an increased ferritin degradation and free iron release from the lysosomes in HK-2 cells following cisplatin treatment (Figure 2, $\mathrm{L}-\mathrm{W}$ ). Taken together, these data indicate that ferroptosis is operative in cisplatin-induced AKI. 
An association between ferroptosis and redox perturbations has been described previously $(17,32)$; it is conceivable that ferroptosis may be modulated by MIOX overexpression in cisplatininduced tubular injury, since oxygen radicals are generated in the MIOX-initiated glucuronate/xylulose pathway (19). MIOX, a proximal tubular-specific enzyme, exacerbates kidney redox injury in multiple pathological states (33). Previous publications revealed that transcriptional, translational, epigenetic, and posttranslational events are involved in the regulation of MIOX overexpression, which can promote ROS generation (14, 15, 33, 34). Our previous work showed that MIOX overexpression accentuates tubular redox injury and apoptosis in cisplatin-induced AKI (8), but its role in ferroptosis remains enigmatic. Consistent with our previous work (8), our present studies revealed that MIOX overexpression was detrimental to cellular homeostasis in cisplatininduced AKI and HK-2 cell injury (Figure 5, A-N, and Figure 9, A-F). Since both ferroptosis and apoptosis are important in cisplatin-induced HK-2 cell death and MIOX modulates apoptosis following cisplatin treatment (8), here the question can be raised of whether MIOX can modulate the processes of ferroptosis. In view of this, we observed that the significant ferroptosis-specific cell death, induced by RSL3, was modulated by MIOX expression profile (Figure 5, O and $\mathrm{P}$ ). Notably, ferroptosis process is somewhat difficult to investigate because of the lack of specific markers. In this regard, pronounced relentless lipid hydroperoxidation and iron overload are regarded as the key characteristics of ferroptosis $(24,25)$. Lipid hydroperoxides, the executioner of ferroptosis, decompose into reactive intermediaries, which then lead to massive cell death (35), while the increased intracellular free iron acts as a catalyst aiding in the execution of ferroptosis (36). In this study, 4-HNE, the end product of lipid hydroperoxides (37), was used to assess the state of lipid hydroperoxidation. Both in vivo and in vitro studies demonstrated that the increase of 4-HNE levels, induced by cisplatin administration, were accentuated by MIOX overexpression while dampened by MIOX gene disruption (Figure 4, E-L, and Figure 9, G-M). In addition, labile iron pool assay revealed an increase in intracellular free iron levels in HK-2 cells following cisplatin treatment, which was regulated by MIOX expression profile (Figure $7 \mathrm{~N}$ ). Taking these data together, one can conclude that ferroptosis process in cisplatin-induced AKI is modulated by MIOX overexpression.

The next question addressed was how MIOX overexpression promotes ferroptosis following cisplatin treatment. Ferritinophagy, a recently coined term, refers to the process by which a massive amount of ferritin undergoes autophagic targeting and lysosomal degradation $(16,38)$. It is a selective autophagy that relies on the guidance of NCOA4 to carry ferritin to the lysosomes, leading to the generation of intracellular free iron $(38,39)$. In this study, cisplatin treatment led to a simultaneous decrease of FTH1 and NCOA4 protein levels in HK-2 cells, which was modulated by MIOX expression profile (Figure 6). In in vivo studies, the expression levels of NOCA4 were decreased following cisplatin treatment (Figure 10, A-F and M). Interestingly, FTH1 was upregulated in cisplatin-treated mice, which may be due to the modulation by an intricate feedback mechanism (Figure 10, G-O). In support of this feedback mechanism are the studies reported by Gao et al., in which the transcription and expression levels of endogenous
FTH1 were upregulated by the increased levels of intracellular free iron during ferroptosis in HT1080 cells (human fibrosarcoma cell line), while there was a simultaneous degradation of the ectopically expressed GFP-FTH1 (21). Intriguingly, MIOX-KO mice had a remarkable baseline decrease in FTH1, possibly due to the lack of MIOX-derived ROS (Figure 10, K-M). To further confirm the modulation of ferritinophagy by MIOX overexpression, lysosome staining with anti-LAMP1 (40) and ferritin costaining with anti-FTH1 were performed in HK-2 cells. As shown in Figure 7, A-D, LAMP1 and FTH1 were colocalized following cisplatin treatment in HK-2 cells, suggesting that cisplatin treatment leads to ferritin uptake by the lysosomes. The colocalization was inhibited by MIOX siRNA while enhanced by MIOX overexpression (Figure 7, $\mathrm{E}-\mathrm{M})$. Since ferritinophagy is a selective autophagy, it is conceivable that lysosomal permeability increases in this process $(41,42)$. Acridine orange (AO) staining revealed an increase in the lysosomal permeability of HK-2 cells following cisplatin treatment, which was regulated by MIOX expression profile (Figure 8, A-F). In addition, the intracellular levels of NADPH, the biomarker of ferroptosis sensitivity (43), was lower in MIOX-overexpressing cells and MIOX-Tg mice even without cisplatin treatment, suggesting that MIOX overexpression alone can sensitize renal tubular cells to ferroptosis (Figure 8, $\mathrm{P}$ and $\mathrm{O}$ ).

Besides promoting ferroptosis via ferritinophagy and lipid hydroperoxidation, MIOX overexpression may also inhibit the "ferroptosis termination system" by downregulating GPX4 activity and intracellular GSH concentration. Normally, the key antioxidase GPX4, together with GSH, catalyzes noxious lipid hydroperoxides into harmless lipid alcohols, leading to the end of ferroptosis (44). Therefore, it is conceivable that GPX4 inhibitors and system $\mathrm{X}_{\mathrm{c}}^{-}$antagonists (related to GSH depletion) can serve as ferroptosis inducers $(45,46)$. Herein, an obvious decline of GPX4 activity was observed in both cisplatin-treated WT mice and MIOX-Tg mice, whereas it was unaffected in cisplatin-treated MIOX-KO mice (Figure 9N). Similarly, GPX4 activity was reduced in in vitro studies, strengthening the argument that the ferroptosis termination system is indeed inhibited (Figure $8 \mathrm{~N}$ ). The above findings along with the reduced $\mathrm{MBB}$ staining are consistent with our previous observations that the GSH depletion and oxidant stress induced by cisplatin treatment are accelerated by MIOX overexpression (8) (Figure 8, G-L and O). Taken together, these observations indicate that MIOX overexpression can promote ferroptosis directly or via the inhibition of the ferroptosis termination system.

In conclusion, the findings of this study indicate that accentuation of ferroptosis in cisplatin-induced AKI can be achieved via modulation of multiple mechanisms in states of overexpression of MIOX, while its gene disruption reduces such a noxious cellular injury. Lastly, these new insights in the pathogenesis of AKI should give impetus to develop therapeutic strategies (MIOX inhibitors?) to ameliorate renal tubular injury.

\section{Methods}

Cell culture studies. The human proximal tubular epithelial cell line HK-2 was purchased from ATCC. Cells were maintained in DMEM, consisting of $10 \% \mathrm{FBS}, 100 \mathrm{U} / \mathrm{mL}$ penicillin, and $100 \mathrm{U} / \mathrm{mL}$ streptomycin. They were maintained in a humidified environment with 
$5 \% \mathrm{CO}_{2}$ at $37^{\circ} \mathrm{C}$. In addition, MIOX-overexpressing HK-2 cells, generated in our laboratory, were also used for various parallel studies. The cells from near-confluent cultures were trypsin-dissociated and seeded onto collagen-coated 6-well plates at a density of $0.5 \times 10^{5}$ to $1 \times 10^{5}$ in a culture medium containing $2 \%$ FBS. They were allowed to attach overnight, and then treated with $20 \mu \mathrm{M}$ cisplatin (SigmaAldrich, catalog P4394) for 4-48 hours. Other chemicals used to treat the cells were as follows: ferrostatin-1 (Fer-1; Sigma-Aldrich, catalog SML0583, ferroptosis inhibitor; $0.4 \mu \mathrm{M}$ ), deferoxamine (SigmaAldrich, catalog D9533, iron chelator; 10-80 $\mu \mathrm{M}$ ), necrostatin-1 (Nec-1; Cayman Chemical, catalog 4311-88-0, necroptosis inhibitor; $50 \mu \mathrm{M}$ ), and Z-VAD(OMe)-FMK (VAD; Cayman Chemical, catalog 187389-52-2, apoptosis inhibitor; $50 \mu \mathrm{M})$. Treated cells were harvested and used for various morphological and biochemical studies. Additional knockdown studies included the use of MIOX siRNA (Origene, catalog SR310776; $50 \mathrm{nM}$ ) and NCOA4 siRNA (Santa Cruz Biotechnology, catalog sc-61117; $50 \mathrm{nM}$ ) in cells transfected with Lipofectamine 2000 reagent (Invitrogen, catalog 11668019).

Animal model system. Eight-week-old male mice in groups of 8 of the following strains were used: CD1, wild type (WT) C57BL/6J, proximal tubular-specific MIOX knockout (MIOX-KO, C57BL/6J background), and proximal tubular-specific MIOX-overexpressing transgenic (MIOX-Tg, C57BL/6J background). The generation of MIOX-Tg and -KO mice has been previously described in our publication (8). AKI was induced with the administration of a single i.p. injection of cisplatin $(20 \mathrm{mg} / \mathrm{kg})$. Control mice received PBS only. For ferroptosis inhibition studies, Fer-1 was administered 45 minutes to 2 hours before the induction of AKI at a dose of $5 \mathrm{mg} / \mathrm{kg}$. The mice were sacrificed 2 hours or 3 days later, and kidney tissues were harvested for various studies. Before sacrifice, the blood and urine samples were collected. Serum creatinine was measured using a QuantiChrom Creatinine assay kit (BioAssay Systems, catalog DICT-500). Urine samples were centrifuged at $5000 \mathrm{~g}$, and supernatants subjected to $15 \%$ SDS-PAGE. The gels were stained with Coomassie Brilliant Blue reagent.

Morphological studies and tubular damage scoring. Four-micrometer-thick tissue sections were heat-deparaffinized and treated with xylene. They were rehydrated with decreasing concentrations of graded series of ethanol. The sections were then successively stained with hematoxylin (3 minutes) and eosin (30 seconds). After a brief wash with deionized-distilled water, the sections were dehydrated with ethanol, treated with xylene, coverslip-mounted, and evaluated. The sections with H\&E staining were used to assess tubular damage scoring, as previously reported $(47,48)$. For each mouse, 100 tubules from 10 different views were evaluated by 2 investigators. Seven parameters were graded as follows: tubular epithelial cell flattening ( 0 or 1$)$, loss of brush border (0 or 1$)$, cell membrane bleb formation (0 or 2$)$, cytoplasmic vacuolization (0 or 1$)$, cell necrosis (0 or 2$)$, interstitial edema (0 or 1 ), and tubular lumen obstruction (0 or 2). The accumulated values ranging from 0 (unaffected) to 10 (severe damage) were considered as indicative of the extent of tubular damage. For PAS staining, the Periodic Acid Schiff Stain Kit (Abcam, catalog ab150680) was used. Briefly, rehydrated sections were immersed in periodic acid solution for 10 minutes. They were then rinsed with distilled water and incubated with Schiff solution for 30 minutes. After washing with distilled water, the sections were stained with hematoxylin for 3 minutes. After another $\mathrm{H}_{2} \mathrm{O}$ wash, Bluing reagent was applied for 30 seconds and slides rewashed, dehydrated, treated with xylene, and coverslip-mounted.
The tissue sections were evaluated for cellular morphology of tubules by light microscopy.

Immunohistochemical studies. Four-micrometer-thick paraffin tissue sections of kidneys, from renal biopsy tissues of 3 ATN patients, were deparaffinized and rehydrated. After heat-induced epitope (antigen) retrieval, the slide sections were washed twice with TBST containing $0.025 \%$ Triton X-100. For blocking of the background staining, the sections were immersed in $3 \%$ BSA in TBST $(137 \mathrm{mM} \mathrm{NaCl}$, $2.7 \mathrm{mM} \mathrm{KCl}, 16.5 \mathrm{mM}$ Tris, $\mathrm{pH}$ 7.4, containing 0.025\% Triton X-100) for 1 hour at $22^{\circ} \mathrm{C}$. Sections were then incubated with MIOX antibody (1:200), NCOA4 antibody (Bethyl Laboratories, catalog A302-272A; 1:100), and FTH1 antibody (Abcam, catalog ab65080; 1:100) in TBST overnight at $4^{\circ} \mathrm{C}$ and rinsed with TBST. Endogenous peroxidase was suppressed by incubation of the sections with $3 \% \mathrm{H}_{2} \mathrm{O}_{2}$ for 15 minutes. The sections were incubated with HRP-conjugated secondary antibody (Vector Laboratories) for 1 hour at $22^{\circ} \mathrm{C}$. After washing with PBS, DAB solution was applied to the sections for 20-35 seconds to develop the peroxidase reaction product. The sections were then immediately washed with deionized distilled water. The sections were counterstained with hematoxylin, dehydrated in graded series of ethanols, coverslip-mounted, and evaluated by light microscopy.

Immunofluorescence studies. Initially, the HK-2 cells that had undergone various treatments were fixed in $4 \%$ paraformaldehyde. They were then permeabilized with $0.25 \%$ Triton X-100 in PBS buffer. They were immersed in $2 \%$ BSA in PBST to block the nonspecific background. The cells were then individually incubated with the following primary antibodies overnight at $4^{\circ} \mathrm{C}$ : anti-NCOA4 (Bethyl Laboratories, catalog A302-272A; 1:100), anti-FTH1 (Abcam, catalog ab65080; 1:100), anti-4-HNE antibody (Abcam, catalog ab46545; 1:100), and anti-LAMP1 (Cell Signaling Technology, catalog 15665S; 1:100). Subsequently, cells were washed with PBS and incubated with secondary antibodies conjugated with FITC. The cells were then coverslip-mounted and examined by fluorescence microscopy. For kidney tissue immunofluorescence, 4 - $\mu$ m-thick cryostat sections were prepared from a different group of experiments. They were washed with PBS and immersed in 2\% BSA to block the nonspecific background. The sections were then incubated with rabbit polyclonal anti-4-HNE, -NCOA4, or -FTH1 primary antibodies for 2 hours at $22^{\circ} \mathrm{C}$. After 3 washes with PBS, secondary antibodies were applied to the sections and incubation extended for another hour at $22^{\circ} \mathrm{C}$. The sections were rewashed with PBS, coverslip-mounted, and examined with a UV microscope equipped with epi-illumination.

Protein expression studies. Immunoblotting procedures were used to evaluate the protein expression in various samples. Briefly, kidney tissues were diced into $1 \mathrm{~mm}^{3}$ and homogenized in RIPA buffer (150 $\mathrm{mM} \mathrm{NaCl}, 1 \% \mathrm{NP}-40,0.5 \%$ deoxycholate, $0.1 \%$ SDS, and $50 \mathrm{mM}$ Tris, $\mathrm{pH}$ 8.0, containing protease inhibitors). Likewise the cells were lysed with RIPA lysis buffer. The homogenates were centrifuged at $10,000 \mathrm{~g}$ for 5 minutes, and supernatants were collected. Protein concentration in the supernatants was measured with a Pierce BCA Protein Assay Kit (catalog 23225). After adjustment of the concentration $(100 \mu \mathrm{g} / 100 \mu \mathrm{L})$ in each of the samples, equal amounts of protein $(20 \mu \mathrm{g})$ were mixed with the SDS loading buffer, boiled for 5 minutes, ice-cooled, and subjected to $10 \%$ or $15 \%$ SDS-PAGE. Following the fractionation of proteins by SDS-PAGE, the proteins were transferred to PVDF membranes by electroblotting procedures. The PVDF membranes were then individually incubated with the follow- 
ing antibodies at the stated dilutions overnight at $4^{\circ} \mathrm{C}$ : anti-MIOX (1:1000), anti-NCOA4 (Bethyl Laboratories, catalog A302-272A; 1:1000), anti-FTH1 (Cell Signaling Technology, catalog 3998S; 1:1000), anti-GPX4 (Cayman Chemical, catalog 10005258; 1:200), anti-4-HNE (Abcam, catalog ab46545; 1:1000), and anti- $\beta$-actin (Thermo Fisher Scientific, catalog A5441; 1:1000). The membranes were washed with TBST buffer (137 mM NaCl, $2.7 \mathrm{mM} \mathrm{KCl,} 16.5 \mathrm{mM}$ Tris, $\mathrm{pH} 7.4$, containing $0.1 \%$ Tween-20) and incubated with secondary antibodies at $22^{\circ} \mathrm{C}$ for 60 minutes. After another wash with TBST, the protein bands in the blots were detected using the ECL chemiluminescence system (Amersham Biosciences).

Gene expression studies. Real-time PCR was used to evaluate the gene expression in various samples. Briefly, approximately $10 \mathrm{mg}$ of kidney tissue was used to isolate RNA, using TRIzol Reagent (Invitrogen, catalog 15-596-026). The RNA was reverse-transcribed into cDNA using a GoScript Reverse Transcription System (Promega). For quantitative PCR, reaction mixture containing 100 ng cDNA, 1 $\mu \mathrm{mol} / \mathrm{L}$ each of forward and reverse primer, $1 \times$ Fast SYBR Green Master Mix, and $2 \mu \mathrm{L}$ of nuclease-free water in a total volume of $10 \mu \mathrm{L}$ was prepared. The reaction mixture was subjected to PCR in an Applied Biosystems Step1Plus Real-Time PCR System Thermocycler. Relative crossing threshold values in comparison with GAPDH were used to calculate the mRNA levels in different samples. The primers used in this study were: GAPDH, forward 5'-GAATACGGCTACAGCAACAGG-3' and reverse 5'-GGTCTGGGATGGAAATTGT-G-3'; $\beta$-actin, forward 5'-AGACCTCTATGCCAACACAGTG-3' and reverse 5'-ACCGATCCACACAGAGTACTTG-3'; FTH1, forward 5'-GCCCTTTGCAACTTCGTCG-3' and reverse 5'-GTGGTAGTTCTGGCGCACTT-3'; transferrin, forward 5'-AACCAGCTCGAAGGCAAGAA-3' and reverse 5'-ACTGCCCGAGAAGAAACTGG-3'; NGAL, forward 5'-GCCCAGGACTCAACTCAGAA-3' and reverse 5'-GACCAGGATGGAGGTGACAT-3'; KIM-1, forward 5'-GGAAGTAAAGGGGGTAGTGGG-3' and reverse 5'-AAGCAGAAGATGGGCATTGC-3'.

Evaluation of cell death and cell morphology in HK-2 cells. TUNEL assay was used to assess the degree of cell death. An In Situ Cell Death Detection Kit (Roche, catalog 11684795910) was used to detect cellular DNA damage. The cells were washed with PBS 3 times and treated with $4 \%$ paraformaldehyde in PBS for 1 hour at $22^{\circ} \mathrm{C}$. The cells were then permeabilized with $0.1 \%$ Triton $\mathrm{X}-100$ in $0.1 \%$ sodium citrate for 2 minutes at $4^{\circ} \mathrm{C}$. Cells were incubated with TUNEL reagent $(450$ $\mu \mathrm{L}$ label solution and $50 \mu \mathrm{L}$ enzyme solution) for 1 hour at $37^{\circ} \mathrm{C}$. Cells were then washed twice with PBS and examined with a UV microscope equipped with epi-illumination. For cell morphology studies, HK-2 cells were examined by phase-contrast light microscopy immediately after cisplatin treatment. In addition, H\&E staining was used to delineate the finer morphological characteristics of the cells as follows. The HK-2 cells were stained with hematoxylin ( 3 minutes) and eosin (45 seconds) after fixation with $4 \%$ paraformaldehyde ( 15 minutes). Cells were then washed and examined with light microscopy.

Assessment of lysosomal permeability, GSH, and mitochondrial ROS in HK-2 cells. The lysosomal permeability in HK-2 cells was determined by acridine orange $(\mathrm{AO})$ staining. Cells were incubated in the growth medium, inclusive of AO $(4 \mu \mathrm{g} / \mathrm{mL}$; Sigma-Aldrich, catalog A6014), and incubation carried out for 20 minutes at $37^{\circ} \mathrm{C}$. The stained cells were washed twice with $3 \%$ FCS in PBS and immediately examined by fluorescence microscopy. For in situ determination of GSH, MBB (20 $\mu$ M; Sigma-Aldrich, catalog B4380) in PBS was used to stain the cells for 15 minutes at $37^{\circ} \mathrm{C}$ after the removal of culture medium. They were coverslip-mounted and photographed immediately. For determination of mitochondrial ROS, dihydroethidium (DHE; $20 \mu \mathrm{M}$; Sigma-Aldrich, catalog D7008) was used. It was included in the PBS to stain the cells for 20 minutes at $37^{\circ} \mathrm{C}$. To ensure the specificity of DHE staining, $100 \mathrm{nM}$ mitoquinone (MitoQ; Focus Biomolecules, catalog 10-1363) was included in the medium to scavenge mitochondrial ROS. Cells were then coverslip-mounted and examined by fluorescence microscopy.

Determination of NADPH levels in kidney tissues and cells. About $15 \mathrm{mg}$ of cortical kidney tissues or approximately $2 \times 10^{6}$ cells with $70 \%-80 \%$ confluence per culture dish $\left(55 \mathrm{~cm}^{2}\right)$ were used to assess the NADPH levels with an NADP/NADPH Assay Kit (Abcam, catalog ab65349). Kidney tissues were homogenized in $200 \mu \mathrm{L}$ NADP/NADPH Extraction Buffer. Likewise the cells were lysed in $200 \mu \mathrm{L} \mathrm{NADP/}$ NADPH Extraction Buffer. The samples were then briefly vortexed and centrifuged at 14,000 $g$ for 5 minutes. The supernatants were passed through spin columns provided in the kit and centrifuged at 10,000 $\mathrm{g}$ for 40 minutes. The filtered samples were then heat-treated at $60^{\circ} \mathrm{C}$ for 30 minutes to decompose NADP. Fifty-microliter standards or samples were added into the individual wells of a 96-well plate, which had pre-added 100- $\mu \mathrm{L}$ Reaction Mix $(98 \mu \mathrm{L}$ NADP Cycling Buffer and 2 $\mu \mathrm{L}$ NADP Cycling Enzyme Mix). Then, $10 \mu \mathrm{L}$ NADPH Developer (provided in the Abcam kit, catalog ab65349) was added into each well and the reaction extended for 2 hours. After the reaction period, colorimetric readings were made at an OD of $450 \mathrm{~nm}$ using a Microplate Reader (Bio-Rad). Finally, the NADPH levels of the samples were calculated by comparison with a standard curve.

Labile iron pool assay. The intracellular free iron levels were measured by labile iron pool assay, as previously described (21). Cells were briefly trypsinized ( 2 minutes) and immediately washed with PBS. Calcein-acetoxymethyl ester $(0.05 \mu \mathrm{M}$; AnaSpec, catalog AS-72126) in PBS was added to the cells and incubated for 15 minutes at $37^{\circ} \mathrm{C}$. After washing with PBS, the cells were divided into 2 aliquots. The cells from the first aliquot were treated with $100 \mu \mathrm{M}$ deferiprone (Sigma-Aldrich, catalog 379409 ) for 1 hour at $37^{\circ} \mathrm{C}$. The second aliquot of cells was left untreated. Flow cytometric reading was made with excitation of calcein at $488 \mathrm{~nm}$ and emission fluorescence at $525 \mathrm{~nm}$. The increase of mean fluorescence in deferiprone-treated cells was compared with that in untreated cells, which represented the amount of intracellular free iron.

MTT assay. HK-2 cells were seeded onto the 96-well plate ( 3000 cells per well). The cells were treated with $20 \mu \mathrm{M}$ cisplatin for 20 hours. The growth medium was removed after treatment, and cells were cultured in $1 \mathrm{mg} / \mathrm{mL}$ Thiazolyl Blue Tetrazolium Bromide (Sigma-Aldrich, catalog M5655) in fresh growth medium for 4 hours. Supernatants were carefully removed from the wells. Then, $150 \mu \mathrm{L}$ DMSO (Thermo Fisher Scientific, catalog BP231-100) was added into each well. The 96-well plates were subjected to orbital shaking for 10 minutes to completely dissolve formazan. The absorbance at $490 \mathrm{~nm}$ wavelength was then measured by a Microplate Reader.

Measurement of GPX4 activity. GPX4 activity was determined as previously described (18). For in vitro studies, approximately $2 \times 10^{6}$ cells from culture dishes $\left(55 \mathrm{~cm}^{2}\right)$ were harvested and homogenized in $200 \mu \mathrm{L}$ of buffer $\left(0.1 \mathrm{M} \mathrm{KH}_{2} \mathrm{PO}_{4} / \mathrm{K}_{2} \mathrm{HPO}_{4}, 0.15 \mathrm{M} \mathrm{KCl}, 0.05 \%\right.$ [wt/ vol] CHAPS, $5 \mathrm{mM} \beta$-mercaptoethanol, and protease inhibitors, $\mathrm{pH}$ 7.4). For in vivo studies, approximately $20 \mathrm{mg}$ kidney tissue, frozen 
in liquid nitrogen, was ground into powder with a pestle and mortar and then homogenized in $200 \mu \mathrm{L}$ buffer. The homogenates were then centrifuged at $15,000 \mathrm{~g}$ for 20 minutes at $4^{\circ} \mathrm{C}$, and the supernatants were saved. An aliquot was used to measure the protein concentration, using Bradford Reagent (Bio-Rad). For GPX4 activity, $50 \mu \mathrm{L}$ of supernatant was added into $1 \mathrm{~mL}$ of assay buffer ( $5 \mathrm{mM}$ EDTA, $5 \mathrm{mM}$ reduced glutathione [GSH; Sigma-Aldrich, catalog 1294820], 0.1\% [vol/vol] Triton X-100, $180 \mathrm{IU} / \mathrm{mL}$ glutathione reductase [SigmaAldrich, catalog G9297], and $160 \mathrm{mM} \mathrm{NADPH} / \mathrm{H}^{+}$[Sigma-Aldrich, catalog 10107824001], pH 7.8). After 5 minutes of incubation at $22^{\circ} \mathrm{C}, 5 \mu \mathrm{L}$ of $30 \mathrm{mM}$ cumene hydroperoxide (Sigma-Aldrich, cata$\log 247502)$ was added to initiate the reaction. The readings were recorded at an absorbance of $340 \mathrm{~nm}$ and measured every 10 seconds until the stability was established, i.e., no detectable change in absorbance. The kinetics representing the rate of change in absorbance, reflective of GPX4 activity, was then calculated.

Spatial distribution of ferrum in the lysosomes. To delineate the localization of ferrum in cisplatin-treated HK-2 cells, first LysoTracker Red (Thermo Fisher Scientific, catalog L7528) in a final concentration of $75 \mathrm{nM}$ was added into the culture medium for 60 minutes. The live cells were immediately photographed to demarcate the fluorescent lysosomes (red fluorescence). Then, the cells were reincubated in 1 $\mathrm{mL} 90 \%$ (vol/vol) ethanol containing $5 \mu \mathrm{L}$ ferrum solution for $15 \mathrm{~min}$ utes at $37^{\circ} \mathrm{C}$. The ferrum solution was prepared by addition of $50 \mu \mathrm{L}$ chloroform into 1 vial of ferrum (Ursa Bioscience, 520-R). The cells were then rephotographed by fluorescence microscopy to detect ferrum (green fluorescence).

Statistics. Two-tailed Student's $t$ test was used for difference analysis of 2 groups, and 1-way ANOVA with Dunn's multiple comparisons was performed for difference analysis of 3 or more groups.
$P$ less than 0.05 was considered significant. Data were expressed as mean \pm SD. GraphPad Prism 7 and Microsoft Excel 2013 were used for all calculations.

Study approval. All animal procedures used in this study were approved by the Animal Care and Use Committee of Northwestern University (2018-2043) and the Second Xiangya Hospital of Central South University, China (2018sydw0276). All human studies were approved by the Second Xiangya Hospital of Central South University (2019-S078). Written informed consent from participants or their guardians was obtained.

\section{Author contributions}

FD contributed to the study design, experiments, data analysis, and manuscript preparation. IS contributed to MIOX overexpression HK-2 cell line preparation and study design. YD contributed to study design and manuscript preparation. MY contributed to collection of human patient samples and clinical data and to research related to CD1 mice. YSK contributed to overall study design, data analysis, and manuscript preparation.

\section{Acknowledgments}

This work is supported by a National Institute of Diabetes and Digestive and Kidney Diseases grant (DK-60635), Visiting Scholarships from China Scholar Council (201706370164), and the National Natural Science Foundation of China (81470925).

Address correspondence to: Yashpal S. Kanwar, Department of Pathology, Northwestern University Medical School, 303 East Chicago Avenue, Chicago, Illinois 60611, USA. Phone: 312.503.0004; Email: y-kanwar@northwestern.edu.
1. Li F, Liu Z, Tang C, Cai J, Dong Z. FGF21 is induced in cisplatin nephrotoxicity to protect against kidney tubular cell injury. FASEB $J$. 2018;32(6):3423-3433.

2. Wang C, et al. Loss of DEPTOR in renal tubules protects against cisplatin-induced acute kidney injury. Cell Death Dis. 2018;9(5):441.

3. Lameire $\mathrm{NH}$, et al. Acute kidney injury: an increasing global concern. Lancet. 2013;382(9887):170-179.

4. Faubel S, et al. Ongoing clinical trials in AKI. Clin J Am Soc Nephrol. 2012;7(5):861-873.

5. Basile DP, et al. Progression after AKI: understanding maladaptive repair processes to predict and identify therapeutic treatments. JAm Soc Nephrol. 2016;27(3):687-697.

6. Vanmassenhove J, Kielstein J, Jörres A, Biesen WV. Management of patients at risk of acute kidney injury. Lancet. 2017;389(10084):2139-2151.

7. Linkermann A, Chen G, Dong G, Kunzendorf U, Krautwald S, Dong Z. Regulated cell death in AKI. J Am Soc Nephrol. 2014;25(12):2689-2701.

8. Dutta RK, Kondeti VK, Sharma I, Chandel NS, Quaggin SE, Kanwar YS. Beneficial effects of $M y o$-inositol oxygenase deficiency in cisplatin-induced AKI. JAm Soc Nephrol. 2017;28(5):1421-1436.

9. Stockwell BR, et al. Ferroptosis: a regulated cell death nexus linking metabolism, redox biology, and disease. Cell. 2017;171(2):273-285.
10. Wang $\mathrm{H}$, et al. Characterization of ferroptosis in murine models of hemochromatosis. Hepatology. 2017;66(2):449-465.

11. Bauckman KA, Mysorekar IU. Ferritinophagy drives uropathogenic Escherichia coli persistence in bladder epithelial cells. Autophagy. 2016;12(5):850-863.

12. Martin-Sanchez D, et al. Ferroptosis, but not necroptosis, is important in nephrotoxic folic acid-induced AKI. J Am Soc Nephrol. 2017;28(1):218-229.

13. Nayak B, et al. Modulation of renal-specific oxidoreductase/myo-inositol oxygenase by high-glucose ambience. Proc Natl Acad Sci U S A. 2005;102(50):17952-17957.

14. Sharma I, Dutta RK, Singh NK, Kanwar YS. High glucose-induced hypomethylation promotes binding of Sp-1 to Myo-inositol oxygenase: implication in the pathobiology of diabetic tubulopathy. Am J Pathol. 2017;187(4):724-739.

15. Sharma I, Tupe RS, Wallner AK, Kanwar YS. Contribution of myo-inositol oxygenase in AGE:RAGE-mediated renal tubulointerstitial injury in the context of diabetic nephropathy. Am JPhysiol Renal Physiol. 2018;314(1):F107-F121.

16. Mancias JD, Wang X, Gygi SP, Harper JW, Kimmelman AC. Quantitative proteomics identifies NCOA4 as the cargo receptor mediating ferritinophagy. Nature. 2014;509(7498):105-109.

17. Skouta R, et al. Ferrostatins inhibit oxidative lipid damage and cell death in diverse disease models. JAm Chem Soc. 2014;136(12):4551-4556.

18. Ingold I, et al. Selenium utilization by GPX 4 is required to prevent hydroperoxide-induced ferroptosis. Cell. 2018;172(3):409-422.e21.

19. Prabhu KS, Arner RJ, Vunta H, Reddy CC. Up-regulation of human myo-inositol oxygenase by hyperosmotic stress in renal proximal tubular epithelial cells. J Biol Chem. 2005;280(20):19895-19901.

20. Andrews NC, Schmidt PJ. Iron homeostasis. Annu Rev Physiol. 2007;69:69-85.

21. Gao M, Monian P, Pan Q, Zhang W, Xiang J, Jiang $\mathrm{X}$. Ferroptosis is an autophagic cell death process. Cell Res. 2016;26(9):1021-1032.

22. Kers J, Leemans JC, Linkermann A. An overview of pathways of regulated necrosis in acute kidney injury. Semin Nephrol. 2016;36(3):139-152.

23. Wenzel SE, et al. PEBP1 wardens ferroptosis by enabling lipoxygenase generation of lipid death signals. Cell. 2017;171(3):628-641.e26.

24. Cao JY, Dixon SJ. Mechanisms of ferroptosis. Cell Mol Life Sci. 2016;73(11-12):2195-2209.

25. Dixon SJ, et al. Ferroptosis: an iron-dependent form of nonapoptotic cell death. Cell. 2012;149(5):1060-1072.

26. Friedmann Angeli JP, et al. Inactivation of the ferroptosis regulator Gpx4 triggers acute renal failure in mice. Nat Cell Biol. 2014;16(12):1180-1191.

27. Linkermann A, et al. Synchronized renal tubular 
cell death involves ferroptosis. Proc Natl Acad Sci US A. 2014;111(47):16836-16841.

28. Hirschhorn T, Stockwell BR. The development of the concept of ferroptosis. Free Radic Biol Med. 2019;133:130-143.

29. Hou W, et al. Autophagy promotes ferroptosis by degradation of ferritin. Autophagy. 2016;12(8):1425-1428.

30. Zarjou A, et al. Proximal tubule H-ferritin mediates iron trafficking in acute kidney injury. J Clin Invest. 2013;123(10):4423-4434.

31. Guo J, et al. Ferroptosis: a novel anti-tumor action for cisplatin. Cancer Res Treat. 2018;50(2):445-460.

32. Totsuka K, et al. Oxidative stress induces ferroptotic cell death in retinal pigment epithelial cells. Exp Eye Res. 2019;181:316-324.

33. Tominaga T, Dutta RK, Joladarashi D, Doi T, Reddy JK, Kanwar YS. Transcriptional and translational modulation of myo-inositol oxygenase (Miox) by fatty acids: implications in renal tubular injury induced in obesity and diabetes. J Biol Chem. 2016;291(3):1348-1367.

34. Zhan M, Usman IM, Sun L, Kanwar YS. Disruption of renal tubular mitochondrial quality control by Myo-inositol oxygenase in diabetic kidney disease. J Am Soc Nephrol. 2015;26(6):1304-1321.
35. Gaschler MM, Stockwell BR. Lipid peroxidation in cell death. Biochem Biophys Res Commun. 2017;482(3):419-425.

36. Gao M, Monian P, Quadri N, Ramasamy R, Jiang $\mathrm{X}$. Glutaminolysis and transferrin regulate ferroptosis. Mol Cell. 2015;59(2):298-308.

37. Ayala A, Muñoz MF, Argüelles S. Lipid peroxidation: production, metabolism, and signaling mechanisms of malondialdehyde and 4-hydroxy-2-nonenal. Oxid Med Cell Longev. 2014;2014:360438.

38. Masaldan S, et al. Iron accumulation in senescent cells is coupled with impaired ferritinophagy and inhibition of ferroptosis. Redox Biol. 2018;14:100-115.

39. Mancias JD, Kimmelman AC. Mechanisms of selective autophagy in normal physiology and cancer. J Mol Biol. 2016;428(9 pt A):1659-1680.

40. Rüder C, et al. The tumor-associated antigen EBAG9 negatively regulates the cytolytic capacity of mouse $\mathrm{CD} 8^{+} \mathrm{T}$ cells. JClin Invest. 2009;119(8):2184-2203.

41. Morris G, Walker AJ, Berk M, Maes M, Puri BK. Cell death pathways: a novel therapeutic approach for neuroscientists. Mol Neurobiol. 2018;55(7):5767-5786.

42. Mancias JD, et al. Ferritinophagy via NCOA4 is required for erythropoiesis and is regulated by iron dependent HERC2-mediated proteolysis. Elife. 2015;4:e10308.

43. Shimada K, Hayano M, Pagano NC, Stockwell BR. Cell-line selectivity improves the predictive power of pharmacogenomic analyses and helps identify NADPH as biomarker for ferroptosis sensitivity. Cell Chem Biol. 2016;23(2):225-235.

44. Kinowaki Y, et al. Glutathione peroxidase 4 overexpression inhibits ROS-induced cell death in diffuse large B-cell lymphoma. Lab Invest. 2018;98(5):609-619.

45. Imai H, Matsuoka M, Kumagai T, Sakamoto T, Koumura T. Lipid peroxidation-dependent cell death regulated by GPx 4 and ferroptosis. Curr Top Microbiol Immunol. 2017;403:143-170.

46. Yu H, Guo P, Xie X, Wang Y, Chen G. Ferroptosis, a new form of cell death, and its relationships with tumourous diseases. JCell Mol Med. 2017;21(4):648-657.

47. Paller MS, Hoidal JR, Ferris TF. Oxygen free radicals in ischemic acute renal failure in the rat. JClin Invest. 1984;74(4):1156-1164.

48. Guo Y, et al. MicroRNA-709 mediates acute tubular injury through effects on mitochondrial function. JAm Soc Nephrol. 2018;29(2):449-461. 